\title{
ERROR ESTIMATES FOR GALERKIN APPROXIMATIONS OF THE "CLASSICAL" BOUSSINESQ SYSTEM
}

\author{
D. C. ANTONOPOULOS AND V. A. DOUGALIS
}

\begin{abstract}
We consider the "classical" Boussinesq system in one space dimension and its symmetric analog. These systems model two-way propagation of nonlinear, dispersive long waves of small amplitude on the surface of an ideal fluid in a uniform horizontal channel. We discretize an initial-boundary-value problem for these systems in space using Galerkin-finite element methods and prove error estimates for the resulting semidiscrete problems and also for their fully discrete analogs effected by explicit Runge-Kutta time-stepping procedures. The theoretical orders of convergence obtained are consistent with the results of numerical experiments that are also presented.
\end{abstract}

\section{INTRODUCTION}

In this paper we will analyze Galerkin approximations to the so-called "classical" Boussinesq system

$$
\begin{aligned}
& \eta_{t}+u_{x}+(\eta u)_{x}=0, \\
& u_{t}+\eta_{x}+u u_{x}-\frac{1}{3} u_{x x t}=0,
\end{aligned}
$$

which is an approximation of the two-dimensional Euler equations of water-wave theory that models two-way propagation of long waves of small amplitude on the surface of an ideal fluid in a uniform horizontal channel of finite depth. The variables in (1.1) are nondimensional and unscaled; $x$ and $t$ are proportional to position along the channel and time, respectively, and $\eta=\eta(x, t)$ and $u=u(x, t)$ are proportional to the elevation of the free surface above a level of rest represented by $\eta=0$, and to the depth-averaged mean horizontal velocity of the fluid.

The system (1.1) is a member of a general family of Boussinesq systems derived in [1] that are approximations to the Euler equations of the same order as (1.1) and whose nonlinear and dispersive terms are of equal importance when written in scaled form. These systems are written as

$$
\begin{aligned}
& \eta_{t}+u_{x}+(\eta u)_{x}+a u_{x x x}-b \eta_{x x t}=0, \\
& u_{t}+\eta_{x}+u u_{x}+c \eta_{x x x}-d u_{x x t}=0,
\end{aligned}
$$

where $a, b, c, d$ are real parameters satisfying $a+b=\frac{1}{2}\left(\theta^{2}-1 / 3\right), c+d=\frac{1}{2}\left(1-\theta^{2}\right)$, where $0 \leq \theta \leq 1$. The specific system (1.1) was previously formally derived from the Euler equations, in the appropriate parameter regime, in [22], 24], and [29]. It has been widely used in the engineering fluid mechanics literature for computations of long, nonlinear dispersive waves, starting with [22] and [23].

Received by the editor August 26, 2010 and, in revised form, October 6, 2011.

2010 Mathematics Subject Classification. Primary 65M60, 35Q53.

Key words and phrases. Boussinesq systems, nonlinear dispersive waves, first-order hyperbolics, Galerkin methods, error estimates. 
Existence and uniqueness of the initial-value problem for (1.1) posed for $x \in \mathbb{R}$ and $t \geq 0$ and supplemented by initial conditions of the form

$$
\eta(x, 0)=\eta_{0}(x), \quad u(x, 0)=u_{0}(x), \quad x \in \mathbb{R},
$$

was studied by Schonbeck, 25] and Amick, 2]. In these papers global existence and uniqueness was established for infinitely differentiable initial data of compact support such that $\eta_{0}(x)>-1, x \in \mathbb{R}$. In 12 the theory of $[25$ and 2 was used to prove that given initial data $\left(\eta_{0}, u_{0}\right) \in H^{s}(\mathbb{R}) \times H^{s+1}(\mathbb{R})$ for $s \geq 1$, such that $\inf _{x \in \mathbb{R}} \eta_{0}(x)>-1$, there is a unique solution $(\eta, u)$ which, for any $T>0$, lies in $C\left(0, T ; H^{s}(\mathbb{R})\right) \times C\left(0, T ; H^{s+1}(\mathbb{R})\right)$. (Here, $H^{s}(\mathbb{R})$ is the usual, $L^{2}$-based Sobolev space of functions on $\mathbb{R}$ and $C(0, T ; X)$ denotes the space of functions $\phi=\phi(t)$ having, for each $t \in[0, T]$, values in a Banach space $X$ and are such that the map $[0, T] \rightarrow\|\phi\|_{X}$ is continuous.)

It is well known that the initial-value problem (1.1), (1.3) has classical solitarywave solutions. In [4 we construct such solutions numerically and investigate, by means of numerical experiments, their properties and interactions. We use fully discrete Galerkin methods that approximate solutions of (1.1) posed on finite intervals and subject to boundary conditions. It is of interest therefore to study initial-boundary-value problems (ibvp's) for (1.1) and establish error estimates for their numerical approximations.

In this paper we shall analyze the numerical solution of the following ibvp for (1.1): For some $0<T<\infty$, we seek $\eta=\eta(x, t), u=u(x, t)$, defined for $0 \leq x \leq 1$, $0 \leq t \leq T$, and satisfying

$$
\begin{aligned}
& \eta_{t}+u_{x}+(\eta u)_{x}=0, \quad(x, t) \in[0,1] \times[0, T], \\
& u_{t}+\eta_{x}+u u_{x}-\frac{1}{3} u_{x x t}=0, \quad(x, t) \in[0,1] \times[0, T], \\
& \eta(x, 0)=\eta_{0}(x), \quad u(x, 0)=u_{0}(x), \quad x \in[0,1], \\
& u(0, t)=0, \quad u(1, t)=0, \quad t \in[0, T] .
\end{aligned}
$$

This ibvp (for $0<t<\infty$ ) has been studied by Adamy, [1, who showed that it has weak (distributional) solutions $(\eta, u) \in L^{\infty}\left(\mathbb{R}^{+} ; L^{1} \times H_{0}^{1}\right)$ provided, e.g., that $\eta_{0} \in L^{1}, u_{0} \in H_{0}^{1}$ with $\inf _{x \in[0,1]} \eta_{0}(x)>-1$. (Here $L^{1}=L^{1}(0,1)$, and $H_{0}^{1}=H_{0}^{1}(0,1)$ is the subspace of the Sobolev space $H^{1}(0,1)$ whose elements vanish at $x=0$ and $x=1$.) The proof uses a parabolic regularization of the first pde of $(\mathrm{CB})$, a technique used in the context of the Cauchy problem by Schonbeck 25]. It should be noted that the homogeneous Dirichlet boundary conditions on $u$ in (CB) are one kind of boundary conditions that lead to well-posed ibvp's in the case of the linearized system; see [19. It is noteworthy that the CB system needs only two boundary conditions for well-posedness as opposed to the four boundary conditions (for example, Dirichlet conditions on $\eta$ and $u$ at each endpoint of the interval) required in the case of other Boussinesq systems, such as the BBM-BBM $(a=c=0, b=d$ in (1.2), cf. [10]), or the Bona-Smith systems $(a=0, b=d>0$, $c<0$ in (1.2), 7.) The case of the homogeneous Dirichlet boundary conditions in (CB) may be viewed as a base for studying the nonhomogeneous analog wherein $u(0, t)$ and $u(1, t)$ are given functions of $t \geq 0$ corresponding to measurements of the velocity variable at two points along the channel.

In [13, Bona, Colin, and Lannes introduced another type of Boussinesq systems that they called "completely symmetric". These, when scaled, are approximations of the Euler equations of the same order and are obtained by a nonlinear change 
of variables from the usual systems (1.2); they have certain mathematical and modelling advantages over the latter. In this paper we shall also therefore consider the analogous problem for the symmetric system, specifically the ibvp

$$
\begin{aligned}
& \eta_{t}+u_{x}+\frac{1}{2}(\eta u)_{x}=0, \quad(x, t) \in[0,1] \times[0, T], \\
& u_{t}+\eta_{x}+\frac{3}{2} u u_{x}+\frac{1}{2} \eta \eta_{x}-\frac{1}{3} u_{x x t}=0, \quad(x, t) \in[0,1] \times[0, T], \\
& \eta(x, 0)=\eta_{0}(x), \quad u(x, 0)=u_{0}(x), \quad x \in[0,1] \\
& u(0, t)=0, \quad u(1, t)=0, \quad t \in[0, T] .
\end{aligned}
$$

It is not hard to see that the solution of (SCB $)$ satisfies the conservation property

$$
\|\eta(t)\|^{2}+\|u(t)\|^{2}+\frac{1}{3}\left\|u_{x}(t)\right\|^{2}=\left\|\eta_{0}\right\|^{2}+\left\|u_{0}\right\|^{2}+\frac{1}{3}\left\|u_{0}^{\prime}\right\|^{2},
$$

for $t \geq 0$, which simplifies the study of its well-posedness and the analysis of its numerical approximations as will be seen in the sequel.

In this paper we shall analyze semidiscrete and fully discrete Galerkin-finite element methods for the ibvp's ( $\mathrm{CB}$ ) and $(\underline{\mathrm{SCB}})$ assuming that their solutions are sufficiently smooth. Previously, rigorous error estimates for Galerkin methods for these "classical" Boussinesq systems were proved in the case of the periodic initialvalue problem in [3] and [9]. (For numerical work for this type and for other Boussinesq systems of the form (1.2) we refer the reader, e.g., to [3], [5], 20], 21], [10, 8], 14, 15], 16, 4].)

The analysis of Galerkin methods for $(\mathrm{CB})$ or $(\mathrm{SCB})$ is of considerable interest due to the loss of optimal order of accuracy that emerges from the error estimates and is also supported by the numerical experiments. We shall investigate this phenomenon in detail in the paper but one may in general say that in the uniform spatial mesh case the (limited) loss of accuracy seems to be due to a combination of effects related to the boundary conditions and the specific form of the "classical" Boussinesq systems. (For example, no loss of accuracy occurs in the case of periodic boundary conditions on $\eta$ and $u$ for these systems; cf. [3], 9.) In the case of general (quasiuniform) mesh the (more severe) loss of accuracy seems to stem from the lack of cancellation effects that are present in the uniform mesh case, and from the hyperbolic character of the first pde of these systems. (The loss of optimal order of accuracy in standard Galerkin semidiscretizations of first-order hyperbolic equations manifested in various contexts is well known and was earlier observed, e.g., by Dupont in [18.)

In section 2 we consider the standard Galerkin semidiscretizations of (CB) and $(\underline{\mathrm{SCB}})$ in the space of (at least) continuous functions on $[0,1]$ that are piecewise polynomials of degree at most $r-1(r \geq 2$, integer) with respect to a quasiuniform mesh. We prove that if the solution $(\eta, u)$ of $(\underline{\mathrm{CB}})$ or $(\underline{\mathrm{SCB}})$ is sufficiently smooth and $\left(\eta_{h}, u_{h}\right)$ is the semidiscrete approximation, then $\left\|\eta-\eta_{h}\right\|=O\left(h^{r-1}\right)$ and $\left\|u-u_{h}\right\|_{1}=O\left(h^{r-1}\right)$. (Here $\|\cdot\|,\|\cdot\|_{1}$ denote, respectively, the $L^{2}$ and $H^{1}$ norms on $[0,1]$.) These rates of convergence are suboptimal in the case of $\eta$ and optimal for $u$; numerical experiments in section 6 indicate that they are sharp.

If the mesh is uniform, better rates are obtained. In section 3 we examine the standard Galerkin semidiscretization of the two systems in the space of continuous, piecewise linear functions and we prove, among other things, that the improved estimates $\left\|\eta-\eta_{h}\right\|=O\left(h^{3 / 2}\right),\left\|u-u_{h}\right\|=O\left(h^{2}\right)$ hold, as a result of a suitable superaccuracy estimate involving the error of the interpolant into the finite element space. In section 4 we consider the case of $C^{2}$ cubic splines. Here, using some 
relevant superconvergence results of Wahlbin [28, allows proving a series of suitable superaccuracy estimates for the error of the interpolant and that of the elliptic projection that lead to error estimates such as $\left\|\eta-\eta_{h}\right\|=O\left(h^{3.5} \sqrt{\ln 1 / h}\right), \| u-$ $u_{h} \|=O\left(h^{4} \sqrt{\ln 1 / h}\right)$. These are consistent with the rates of convergence of the errors obtained from numerical experiments presented in section 6 .

In section 5 we turn to the analysis of fully discrete schemes. We consider only explicit time-stepping schemes in order to avoid the more costly implicit methods that require solving nonlinear systems of equations at every time step. Of course, with explicit methods there arises the issue of stability. We confine ourselves to a uniform mesh spatial discretization and consider three representative explicit Runge-Kutta schemes, namely, the Euler, the improved Euler, and the classical, four-stage, Runge-Kutta methods, whose orders of accuracy are 1, 2, and 4, respectively. We couple the Euler and improved Euler schemes with a piecewise linear spatial discretization and the fourth-order RK scheme with cubic splines. We show that the stability restrictions on the time step $k$ needed by these schemes are of the form $k=O\left(h^{2}\right), k=O\left(h^{4 / 3}\right)$, and $k \leq \lambda_{0} h$ for a constant $\lambda_{0}$ sufficiently small, for the schemes with Euler, improved Euler, and fourth-order RK temporal discretizations, respectively. Under these restrictions, we prove optimal-order in time error estimates; the spatial rates of convergence are these of the semidiscrete approximations.

In this paper we let $C^{k}=C^{k}[0,1], k=0,1,2, \ldots$, denote the space of $k$ times continuously differentiable functions on $[0,1]$ and define $C_{0}^{k}=\left\{\phi \in C^{k} ; \phi(0)=\right.$ $\phi(1)=0\}$. For integer $k \geq 0$ we let $H^{k},\|\cdot\|_{k}$ denote the usual $L^{2}$-based Sobolev space of classes of functions on $[0,1]$ and its associated norm. (In the case $k=1$ we use the equivalent norm defined by $\|v\|_{1}=\left(\|v\|^{2}+\frac{1}{3}\left\|v^{\prime}\right\|^{2}\right)^{1 / 2}$.) The inner product and norm on $L^{2}=L^{2}(0,1)$ is denoted simply by $\|\cdot\|,(\cdot, \cdot)$, respectively, while the norms on $L^{\infty}=L^{\infty}(0,1)$ and on $W_{\infty}^{k}=W_{\infty}^{k}(0,1)$ by $\|\cdot\|_{\infty},\|\cdot\|_{k, \infty}$. We let $\mathbb{P}_{r}$ be the polynomials of degree $\leq r$. By $\langle\cdot, \cdot\rangle,|\cdot|$, we shall denote the Euclidean inner product and norm on $\mathbb{R}^{n}$.

In http://arxiv.org/abs/1008.4248 the interested reader may find an extended version of the present paper ([6] $)$ including additional results as well as details of proofs omitted herein.

\section{Standard Galerkin SEMidiscretization on A QUASIUniform MeSh}

Let $0=x_{1}<x_{2}<\cdots<x_{N+1}=1$ denote a quasiuniform partition of [0,1] with $h:=\max _{i}\left(x_{i+1}-x_{i}\right)$. Given integers $r \geq 2$ and $0 \leq \mu \leq r-2$, let $S_{h}:=\left\{\phi \in C^{\mu}\right.$ : $\left.\left.\phi\right|_{\left[x_{j}, x_{j+1}\right]} \in \mathbb{P}_{r-1}, 1 \leq j \leq N\right\}, S_{h, 0}=\left\{\phi \in S_{h}, \phi(\overline{0})=\phi(1)=0\right\}$. It is well known that there exists a constant $C$ independent of $h$ such that

$$
\min _{\chi \in S_{h}}\left\{\|w-\chi\|+h\|w-\chi\|_{1}\right\} \leq C h^{r}\|w\|_{r},
$$

for $w \in H^{r}$, and that a similar estimate holds in $S_{h, 0}$ if $w \in H^{r} \cap H_{0}^{1}$. (In what follows, $C$ will denote a generic constant, independent of $h$.) Let $a(\cdot, \cdot)$ denote the bilinear form $a(\psi, \chi):=(\psi, \chi)+\frac{1}{3}\left(\psi^{\prime}, \chi^{\prime}\right) \forall \psi, \chi \in S_{h, 0}$, and let $R_{h}: H^{1} \rightarrow S_{h, 0}$ be the elliptic projection operator relative to $a(\cdot, \cdot)$, defined by $a\left(R_{h} w, \chi\right)=a(w, \chi)$ $\forall \chi \in S_{h, 0}$. It follows by standard estimates that for $k=0,1$,

$$
\left\|R_{h} w-w\right\|_{k} \leq C h^{r-k}\|w\|_{r} \quad \text { if } \quad w \in H^{r} \cap H_{0}^{1} .
$$


It also holds $([17)$ that

$$
\left\|R_{h} w-w\right\|_{\infty}+h\left\|R_{h} w-w\right\|_{1, \infty} \leq C h^{r}\|w\|_{r, \infty},
$$

provided that $w \in W^{r, \infty} \cap H_{0}^{1}$. In addition, the inverse inequalities

$$
\begin{gathered}
\|\chi\|_{1} \leq C h^{-1}\|\chi\|, \\
\|\chi\|_{\infty} \leq C h^{-1 / 2}\|\chi\|,
\end{gathered}
$$

hold for any $\chi \in S_{h}$ (or $\chi \in S_{h, 0}$ ), and so does the estimate, [17,

$$
\|P v-v\|_{\infty} \leq C h^{r}\|v\|_{r, \infty}, \quad \text { for } \quad v \in W^{r, \infty},
$$

where $P: L^{2} \rightarrow S_{h}$ is the $L^{2}$-projection operator onto $S_{h}$.

The standard Galerkin semidiscretization on $S_{h}$ of $(\mathrm{CB})$ is defined as follows: We seek $\eta_{h}:[0, T] \rightarrow S_{h}, u_{h}:[0, T] \rightarrow S_{h, 0}$, such that for $t \in[0, T]$,

$$
\begin{array}{ll}
\left(\eta_{h t}, \phi\right)+\left(u_{h x}, \phi\right)+\left(\left(\eta_{h} u_{h}\right)_{x}, \phi\right)=0 & \forall \phi \in S_{h}, \\
a\left(u_{h t}, \chi\right)+\left(\eta_{h x}, \chi\right)+\left(u_{h} u_{h x}, \chi\right)=0 & \forall \chi \in S_{h, 0},
\end{array}
$$

with initial conditions

$$
\eta_{h}(0)=P \eta_{0}, \quad u_{h}(0)=R_{h} u_{0} .
$$

Similarly, we define the analogous semidiscretization of ( $\underline{\mathrm{SCB}}$ ), which is given for $0 \leq t \leq T$ by

$$
\begin{aligned}
\left(\eta_{h t}, \phi\right)+\left(u_{h x}, \phi\right)+\frac{1}{2}\left(\left(\eta_{h} u_{h}\right)_{x}, \phi\right)=0 & \forall \phi \in S_{h}, \\
a\left(u_{h t}, \chi\right)+\left(\eta_{h x}, \chi\right)+\frac{3}{2}\left(u_{h} u_{h x}, \chi\right)+\frac{1}{2}\left(\eta_{h} \eta_{h x}, \chi\right)=0 & \forall \chi \in S_{h, 0},
\end{aligned}
$$

with

$$
\eta_{h}(0)=P \eta_{0}, \quad u_{h}(0)=R_{h} u_{0} .
$$

Upon choosing a basis for $S_{h}$, it is seen that the semidiscrete problems (2.7), (2.8) and (2.9), (2.10) represent initial-value problems for systems of ode's. Clearly, these systems have unique solutions at least locally in time. One conclusion of the next proposition is that they possess unique solutions up to $t=T$, where $[0, T]$ will denote henceforth the interval of existence of solutions of ([CB) or (SCB $)$.

Proposition 2.1. Let $h$ be sufficiently small. Suppose that the solutions of (CB), and $(\mathrm{SCB})$, are such that $\eta \in C\left(0, T ; W_{\infty}^{r}\right), u \in C\left(0, T ; W_{\infty}^{r} \cap H_{0}^{1}\right)$. Then, the semidiscrete problems (2.7), (2.8) and (2.9), (2.10) have unique solutions $\left(\eta_{h}, u_{h}\right)$ for $0 \leq t \leq T$ that satisfy

$$
\begin{gathered}
\max _{0 \leq t \leq T}\left\|\eta(t)-\eta_{h}(t)\right\| \leq C h^{r-1}, \\
\max _{0 \leq t \leq T}\left\|u(t)-u_{h}(t)\right\|_{1} \leq C h^{r-1} .
\end{gathered}
$$

Proof. We first consider the case of the symmetric system (SCB). Putting $\phi=\eta_{h}$ and $\chi=u_{h}$ in (2.9) and adding the resulting equations, we obtain the discrete analog of (1.4), i.e., that

$$
\left\|\eta_{h}(t)\right\|^{2}+\left\|u_{h}(t)\right\|_{1}^{2}=\left\|\eta_{h}(0)\right\|^{2}+\left\|u_{h}(0)\right\|_{1}^{2}
$$

is valid in the temporal interval of existence of solutions of (2.9)-(2.10). By standard ode theory we conclude that the system (2.9), (2.10) possesses a unique solution in any finite temporal interval $[0, T]$. 
We now let $\rho:=\eta-P \eta, \theta:=P \eta-\eta_{h}, \sigma:=u-R_{h} u, \xi:=R_{h} u-u_{h}$. Using (SCB) and (2.9), (2.10) we obtain for $0 \leq t \leq T$,

$$
\left(\theta_{t}, \phi\right)+\left(\sigma_{x}+\xi_{x}, \phi\right)+\frac{1}{2}\left(\left(\eta u-\eta_{h} u_{h}\right), \phi\right)=0 \quad \forall \phi \in S_{h},
$$

$$
a\left(\xi_{t}, \chi\right)+\left(\rho_{x}+\theta_{x}, \chi\right)+\frac{3}{2}\left(u u_{x}-u_{h} u_{h x}, \chi\right)+\frac{1}{2}\left(\eta \eta_{x}-\eta_{h} \eta_{h x}, \chi\right)=0 \quad \forall \chi \in S_{h, 0} .
$$

Note first that $\eta u-\eta_{h} u_{h}=u(\rho+\theta)-(\rho+\theta)(\sigma+\xi)+\eta(\sigma+\xi), u u_{x}-u_{h} u_{h x}=$ $(u \sigma)_{x}+(u \xi)_{x}-(\sigma \xi)_{x}-\sigma \sigma_{x}-\xi \xi_{x}, \eta \eta_{x}-\eta_{h} \eta_{h x}=(\eta \rho)_{x}+(\eta \theta)_{x}-(\rho \theta)_{x}-\rho \rho_{x}-\theta \theta_{x}$. Now, by continuity, in view of (2.10), we conclude that there exists a maximal temporal instance $t_{h}>0$ such that $\|\theta(t)\|_{\infty} \leq 1$ for $t \leq t_{h}$. Suppose that $t_{h}<T$. Then, taking $\phi=\theta$ in (2.14) and using (2.1)-(2.4), (2.6), and integrating by parts, we have for $0 \leq t \leq t_{h}$,

$$
\begin{aligned}
\frac{1}{2} \frac{d}{d t}\|\theta\|^{2}= & -\left(\sigma_{x}, \theta\right)-\left(\xi_{x}, \theta\right)-\frac{1}{2}\left[\left((\rho u)_{x}, \theta\right)+\frac{1}{2}\left(u_{x} \theta, \theta\right)-\left((\rho \sigma)_{x}, \theta\right)\right. \\
& -\left((\rho \xi)_{x}, \theta\right)-\frac{1}{2}\left(\sigma_{x} \theta, \theta\right)-\frac{1}{2}\left(\xi_{x} \theta, \theta\right)+\left((\eta \sigma)_{x}, \theta\right)+\left((\eta \xi)_{x}, \theta\right] \\
\leq & \left\|\sigma_{x}\right\|\|\theta\|+\left\|\xi_{x}\right\|\|\theta\|+\frac{1}{2}\|u\|_{\infty}\left\|\rho_{x}\right\|\|\theta\|+\frac{1}{2}\left\|u_{x}\right\|_{\infty}\|\rho\|\|\theta\| \\
& +\frac{1}{4}\left\|u_{x}\right\|_{\infty}\|\theta\|^{2}+\frac{1}{2}\left\|\rho_{x}\right\|\|\sigma\|_{\infty}\|\theta\| \\
& +\frac{1}{2}\|\rho\|\left\|\sigma_{x}\right\|_{\infty}\|\theta\|+\frac{1}{2}\|\rho\|_{\infty}\left\|\xi_{x}\right\|\|\theta\| \\
& +C\left\|\rho_{x}\right\|\|\xi\|_{1}\|\theta\|+\frac{1}{4}\left\|\sigma_{x}\right\|_{\infty}\|\theta\|^{2}+\frac{1}{4}\left\|\xi_{x}\right\|\|\theta\|+\frac{1}{2}\|\eta\|_{\infty}\left\|\sigma_{x}\right\|\|\theta\| \\
& +\frac{1}{2}\left\|\eta_{x}\right\|_{\infty}\|\sigma\|\|\theta\|+\frac{1}{2}\|\eta\|_{\infty}\left\|\xi_{x}\right\|\|\theta\|+\frac{1}{2}\left\|\eta_{x}\right\|_{\infty}\|\xi\|\|\theta\| \\
\leq & C\left(h^{r-1}+\|\xi\|_{1}+\|\theta\|\right)\|\theta\|,
\end{aligned}
$$

where $C$ is independent of $t_{h}$. In addition, putting $\chi=\xi$ in (2.15) we similarly obtain, for $0 \leq t \leq t_{h}$,

$$
\begin{aligned}
\frac{1}{2} \frac{d}{d t}\|\xi\|_{1}^{2}= & \left(\rho+\theta, \xi_{x}\right)+\frac{3}{2}\left[\left(u \sigma, \xi_{x}\right)+\left(u \xi, \xi_{x}\right)-\left(\sigma \xi, \xi_{x}\right)+\left(\sigma \sigma_{x}, \xi\right)\right] \\
& +\frac{1}{2}\left[\left(\eta \rho, \xi_{x}\right)+\left(\eta \theta, \xi_{x}\right)-\left(\rho \theta, \xi_{x}\right)-\frac{1}{2}\left(\rho \xi_{x}, \rho\right)-\left(\theta \xi_{x}, \theta\right)\right] \\
\leq & \|\rho\|\left\|\xi_{x}\right\|+\|\theta\|\left\|\xi_{x}\right\|+\frac{3}{2}\|u\|_{\infty}\|\sigma\|\left\|\xi_{x}\right\|+\frac{3}{2}\|u\|_{\infty}\|\xi\|\left\|\xi_{x}\right\| \\
& +\frac{3}{2}\|\sigma\|_{\infty}\|\xi\|\left\|\xi_{x}\right\|+\frac{3}{2}\|\sigma\|_{\infty}\left\|\sigma_{x}\right\|\|\xi\|+\frac{1}{2}\|\eta\|_{\infty}\|\rho\|\left\|\xi_{x}\right\| \\
& +\frac{1}{2}\|\eta\|_{\infty}\|\theta\|\left\|\xi_{x}\right\|+\frac{1}{2}\|\rho\|\left\|\xi_{x}\right\|+C\|\rho\|_{1}\|\rho\|\|\xi\|_{1}+\frac{1}{2}\left\|\xi_{x}\right\|\|\theta\| \\
\leq & C\left(h^{r}+\|\xi\|_{1}+\|\theta\|\right)\|\xi\|_{1} .
\end{aligned}
$$

From (2.16) and (2.17) it is seen that for $0 \leq t \leq t_{h}$ there holds $\frac{d}{d t}\left(\|\theta\|+\|\xi\|_{1}\right) \leq$ $C\left(h^{r-1}+\|\theta\|+\|\xi\|_{1}\right)$, from which, by Gronwall's Lemma and (2.10), we conclude that

$$
\|\theta(t)\|+\|\xi(t)\|_{1} \leq C h^{r-1}, \quad 0 \leq t \leq t_{h}
$$

where $C$ is independent of $t_{h}$. Since by (2.5) $\|\theta\|_{\infty} \leq C h^{-1 / 2}\|\theta\|$, if $h$ is sufficiently small the maximality property of $t_{h}$ is contradicted. Therefore we may take $t_{h}=T$, and (2.11) and (2.12) follow from (2.18), (2.1) and (2.2). In the case of (CB) the invariance property (2.13) no longer holds, and the ivp (2.7), (2.8) has a local unique solution. Using the same notation as in the case of ( $(\underline{\mathrm{SCB}})$, we consider the ivp of 
finding $\theta(t) \in S_{h}, \xi(t) \in S_{h, 0}$ for $t \geq 0$, such that

$$
\begin{aligned}
\left(\theta_{t}, \phi\right)+\left(\sigma_{x}+\xi_{x}, \phi\right)+ & ([u(\rho+\theta)-(\rho+\theta)(\sigma+\xi) \\
+ & \eta(\sigma+\xi)], \phi)=0 \quad \forall \phi \in S_{h}, \\
a\left(\xi_{t}, \chi\right)+\left(\rho_{x}+\theta_{x}, \chi\right)- & \left(u(\sigma+\xi)-\sigma \xi, \chi^{\prime}\right) \\
& -\left(\sigma \sigma_{x}+\xi \xi_{x}, \chi\right)=0 \quad \forall \chi \in S_{h, 0}, \\
\theta(0)= & 0, \quad \xi(0)=0 .
\end{aligned}
$$

Obviously, (2.19) has a local unique solution. Let $t_{h} \in(0, T)$ be the maximal time instance for which this solution exists and satisfies $\|\theta(t)\|_{\infty} \leq 1$ for $0 \leq t \leq t_{h}$. Then, as in the case of ( $\underline{\mathrm{SCB}})$, we obtain again that $\|\theta(t)\|+\|\xi(t)\|_{1} \leq C h^{r-1}$, $0 \leq t \leq t_{h}$, where $C$ is independent of $t_{h}$. We conclude that we may take $t_{h}=T$. If $\eta_{h}=P \eta-\theta, u_{h}=R_{h} u-\xi$, it follows that $\left(\eta_{h}, u_{h}\right)$ is a unique solution of (2.7), (2.8) in $[0, T]$ and that it satisfies the estimates (2.11) and (2.12).

The error estimates of this proposition indicate that the $L^{2}$ rate of convergence that we obtain for the error $\eta-\eta_{h}$ is suboptimal while that of the $H^{1}$ norm of $u-u_{h}$ is optimal. This is consistent with the results of numerical experiments to be presented in section 6 .

\section{Semidiscretization With PieCEWise Linear, CONTINUOUS FUnCtions ON A UNIFORM MESH}

For integer $N \geq 2$ we let $h=1 / N$ and $x_{i}=(i-1) h, i=1,2, \ldots, N+1$, $S_{h}^{2}:=\left\{\phi \in C^{0}:\left.\phi\right|_{\left[x_{j}, x_{j+1}\right]} \in \mathbb{P}_{1}, 1 \leq j \leq N\right\}, S_{h, 0}^{2}=\left\{\phi \in S_{h}^{2}, \phi(0)=\phi(1)=0\right\}$. We will use the operators $P$ and $R_{h}$ introduced in the previous section as well as the estimates (2.1)-(2.6) for $r=2$, putting $S_{h}=S_{h}^{2}, S_{h, 0}=S_{h, 0}^{2}$. In addition, we let $I_{h}, I_{h, 0}$ denote the interpolation operators with respect to the mesh $\left\{x_{j}\right\}$ into the spaces $S_{h}^{2}, S_{h, 0}^{2}$, respectively. It is well known that

$$
\left\|w-I_{h} w\right\|+h\left\|\left(w-I_{h} w\right)^{\prime}\right\| \leq C h^{k}\left\|w^{(k)}\right\|,
$$

holds for $w \in H^{k}, k=1,2$, and that a similar estimate holds in $S_{h, 0}^{2}$ if $w \in H^{k} \cap H_{0}^{1}$. We let $\left\{\phi_{j}\right\}_{j=1}^{N+1}$ denote the basis of $S_{h}^{2}$ satisfying $\phi_{j}\left(x_{i}\right)=\delta_{i j}, 1 \leq i, j \leq N+1$. In the following lemma we collect results that will prove useful in the error estimates that follow.

Lemma 3.1. (i) Let $G_{i j}=\left(\phi_{j}, \phi_{i}\right), 1 \leq i, j \leq N+1$. Then there exist positive constants $c_{1}$ and $c_{2}$ such that $c_{1} h|\gamma|^{2} \leq\langle G \gamma, \gamma\rangle \leq c_{2} h|\gamma|^{2} \forall \gamma \in \mathbb{R}^{N+1}$.

(ii) Let $b \in \mathbb{R}^{N+1}, \gamma=G^{-1} b$, and $\psi=\sum_{j=1}^{N+1} \gamma_{j} \phi_{j}$. Then $\|\psi\| \leq\left(c_{1} h\right)^{-1 / 2}|b|$.

(iii) Let $w \in C^{3}$. Then, there exists a constant $C_{1}=C_{1}\left(\left\|w^{(3)}\right\|_{\infty}\right)$ such that for any $\hat{x} \in\left[x_{i}, x_{i+1}\right]$,

$$
\left(w-I_{h} w\right)(x)=-\frac{1}{2} w^{\prime \prime}(\hat{x})\left(x-x_{i}\right)\left(x_{i+1}-x\right)+\tilde{g}(x), \quad x_{i} \leq x \leq x_{i+1},
$$

where $\|\tilde{g}\|_{\infty}+h\left\|\tilde{g}^{\prime}\right\|_{\infty} \leq C_{1} h^{3}$.

Proof. The proofs of $(i),(i i),($ iii $)$ are given in [18] for continuous, piecewise linear, periodic functions on $[0,1]$. It is straightforward to adapt them in the case of $S_{h}^{2}$ at hand.

The following superapproximation result for the interpolation error, a consequence of cancellations due to the uniform mesh, is central for the sequel. 
Lemma 3.2. Let $v \in C^{3}$ and $w \in C^{1}$. If $\varepsilon:=v-I_{h} v$ and $\psi \in S_{h}^{2}$ is such that $(\psi, \phi)=\left((w \varepsilon)^{\prime}, \phi\right) \forall \phi \in S_{h}^{2}$, then $\|\psi\| \leq C h^{3 / 2}$. If in addition $w(0)=w(1)=0$, then $\|\psi\| \leq C h^{2}$.

Proof. Let $b_{i}:=\left((w \varepsilon)^{\prime}, \phi_{i}\right)=-\left(w \varepsilon, \phi_{i}^{\prime}\right), 1 \leq i \leq N+1$. Clearly, $\left|b_{1}\right|=O\left(h^{2}\right)$ and $\left|b_{N+1}\right|=O\left(h^{2}\right)$. Let $2 \leq i \leq N$. Then, from Lemma 3.1(iii) we have

$$
\begin{aligned}
b_{i}= & \frac{v^{\prime \prime}\left(x_{i}\right)}{2 h} \int_{x_{i-1}}^{x_{i}} w(x)\left(x-x_{i-1}\right)\left(x_{i}-x\right) d x \\
& \quad-\frac{v^{\prime \prime}\left(x_{i}\right)}{2 h} \int_{x_{i}}^{x_{i+1}} w(x)\left(x-x_{i}\right)\left(x_{i+1}-x\right) d x+O\left(h^{3}\right) \\
= & \frac{-v^{\prime \prime}\left(x_{i}\right)}{2 h} \int_{x_{i}}^{x_{i+1}}[w(x)-w(x-h)]\left(x-x_{i}\right)\left(x_{i+1}-x\right) d x+O\left(h^{3}\right) .
\end{aligned}
$$

Therefore, $b_{i}=O\left(h^{3}\right), 2 \leq i \leq N$; consequently $|b|=O\left(h^{2}\right)$ and $\|\psi\|=O\left(h^{3 / 2}\right)$ by Lemma 3.1(ii). If $w(0)=0$, Lemma 3.1( $i i i)$ gives

$$
b_{1}=-\left(w \varepsilon, \phi_{1}^{\prime}\right)=\frac{-v^{\prime \prime}(0)}{2 h} \int_{0}^{h} w(x) x(h-x) d x+O\left(h^{3}\right)=O\left(h^{3}\right) .
$$

Similarly, if $w(1)=0$, then $b_{N+1}=O\left(h^{3}\right)$. Hence, $|b|=O\left(h^{5 / 2}\right)$, giving $\|\psi\|=$ $O\left(h^{2}\right)$ by Lemma $3.1(i i)$.

In the uniform mesh case at hand, and with $S_{h}=S_{h}^{2}, S_{h, 0}=S_{h, 0}^{2}$, we consider again the semidiscretizations (2.7) and (2.9) of ( $(\mathrm{CB})$ and (SCB $)$, respectively, with the initial conditions for both systems now given by the interpolants of $\eta_{0}, u_{0}$ :

$$
\eta_{h}(0)=I_{h} \eta_{0}, \quad u_{h}(0)=I_{h, 0} u_{0} .
$$

The main result of this section is

Theorem 3.1. Let $h=1 / N$ be sufficiently small. Suppose that the solutions of ( $(\mathrm{CB})$ and $(\underline{\mathrm{SCB}})$ are such that $\eta \in C\left(0, T ; C^{3}\right), \eta_{t} \in C\left(0, T ; C^{2}\right), u, u_{t} \in$ $C\left(0, T ; C_{0}^{3}\right)$. Then, the semidiscrete problems (2.7), (3.2) and (2.9), (3.2) have unique solutions $\left(\eta_{h}, u_{h}\right)$ for $0 \leq t \leq T$ that satisfy

(i) $\max _{0 \leq t \leq T}\left\|\eta(t)-\eta_{h}(t)\right\| \leq C h^{3 / 2}, \quad \max _{0 \leq t \leq T}\left\|u(t)-u_{h}(t)\right\|_{1} \leq C h$,

(ii) $\max _{0 \leq t \leq T}\left\|u(t)-u_{h}(t)\right\| \leq C h^{2}, \quad \max _{0 \leq t \leq T}\left\|u_{t}(t)-u_{h t}(t)\right\| \leq C h^{2}$.

Proof. We give the proof in detail in the case of ( $(\underline{\mathrm{SCB}})$, where existence of solutions of the ivp (2.9), (3.2) for $0 \leq t \leq T$ follows from (2.13). The proof in the case of (CB) follows from an argument analogous to that given in the proof of Proposition 2.1 and will be omitted. Let $\rho:=\eta-I_{h} \eta, \theta:=I_{h} \eta-\eta_{h}, \sigma:=u-I_{h, 0} u, \xi:=I_{h, 0} u-u_{h}$. Note that $\eta u-\eta_{h} u_{h}=\eta \sigma+u \theta-\theta \xi+F$, where $F:=\eta \xi+u \rho-\rho \sigma-\rho \xi-\theta \sigma$. In addition, $\eta \eta_{x}-\eta_{h} \eta_{h x}=-\theta \theta_{x}+(\eta \theta)_{x}+G_{x}$, where $G:=\eta \rho-\rho \theta-\frac{1}{2} \rho^{2}$ and $u u_{x}-u_{h} u_{h x}=H_{x}$, where $H:=u \sigma+u \xi-\sigma \xi-\frac{1}{2} \sigma^{2}-\frac{1}{2} \xi^{2}$. Then, from (SCB) and (2.9), (3.2) it follows for $0 \leq t \leq T$ that

$$
\begin{gathered}
\left(\theta_{t}, \phi\right)+\left(\xi_{x}, \phi\right)+\left(\left(\left(1+\frac{1}{2} \eta\right) \sigma\right)_{x}, \phi\right)+\frac{1}{2}\left((u \theta)_{x}, \phi\right)-\frac{1}{2}\left((\theta \xi)_{x}, \phi\right) \\
+\frac{1}{2}\left(F_{x}, \phi\right)=-\left(\rho_{t}, \phi\right) \quad \forall \phi \in S_{h}^{2}, \\
a\left(\xi_{t}, \chi\right)+\left(\theta_{x}, \chi\right)+\left(\rho_{x}, \chi\right)-\frac{1}{2}\left(\theta \theta_{x}, \chi\right)+\frac{1}{2}\left((\eta \theta)_{x}, \chi\right)+\frac{1}{2}\left(G_{x}, \chi\right) \\
+\frac{3}{2}\left(H_{x}, \chi\right)=-\left(\sigma_{t}, \chi\right) \quad \forall \chi \in S_{h, 0}^{2},
\end{gathered}
$$


with

$$
\theta(0)=0, \quad \xi(0)=0 .
$$

(In the right-hand side of (3.4) we used the fact that for $\chi \in S_{h, 0}^{2} a\left(\sigma_{t}, \chi\right)=\left(\sigma_{t}, \chi\right)$, since $\left(v^{\prime}-\left(I_{h, 0} v\right)^{\prime}, \chi^{\prime}\right)=0$ for $v \in H_{0}^{1}$.) In order to show the estimates in $(i)$, we put $\phi=\theta$ and $\chi=\xi$ in (3.3) and (3.4), integrate by parts, and add the resulting equations to get for $0 \leq t \leq T$,

$$
\begin{aligned}
\frac{1}{2} \frac{d}{d t}\left(\|\theta\|^{2}\right. & \left.+\|\xi\|_{1}^{2}\right)+\left(\left(\left(1+\frac{1}{2} \eta\right) \sigma\right)_{x}, \theta\right)+\frac{1}{2}\left((u \theta)_{x}, \theta\right)+\frac{1}{2}\left(F_{x}, \theta\right) \\
& +\left(\rho_{x}, \xi\right)+\frac{1}{2}\left((\eta \theta)_{x}, \xi\right)+\frac{1}{2}\left(G_{x}, \xi\right)+\frac{3}{2}\left(H_{x}, \xi\right)=-\left(\rho_{t}, \theta\right)-\left(\sigma_{t}, \xi\right) .
\end{aligned}
$$

Now, using the approximation properties of $S_{h}^{2}$ and $S_{h, 0}^{2}$, integration by parts, and Lemmas 3.1 and 3.2 we see that

$$
\begin{gathered}
\left|\left(\left(\left(1+\frac{1}{2} \eta\right) \sigma\right)_{x}, \theta\right)\right|=\left|\left(P\left[\left(\left(1+\frac{1}{2} \eta\right) \sigma\right)_{x}\right], \theta\right)\right| \leq C h^{3 / 2}\|\theta\|, \\
\left|\left((u \theta)_{x}, \theta\right)\right|=\frac{1}{2}\left|\left(u_{x} \theta, \theta\right)\right| \leq C\|\theta\|^{2}, \\
\left|\left(F_{x}, \theta\right)\right| \leq\left|\left((\eta \xi)_{x}, \theta\right)\right|+\left|\left((u \rho)_{x}, \theta\right)\right|+\left|\left((\rho \sigma)_{x}, \theta\right)\right|+\left|\left((\rho \xi)_{x}, \theta\right)\right|+\frac{1}{2}\left|\left(\sigma_{x} \theta, \theta\right)\right| \\
\leq C\left(\|\xi\|_{1}\|\theta\|+h^{2}\|\theta\|+h\|\theta\|^{2}\right), \\
\left|\left(\rho_{x}, \xi\right)\right| \leq C h^{2}\|\xi\|_{1}, \quad\left|\left((\eta \theta)_{x}, \xi\right)\right| \leq C\|\xi\|_{1}\|\theta\|, \\
\left|\left(G_{x}, \xi\right)\right| \leq\left|\left(\eta \rho, \xi_{x}\right)\right|+\left|\left(\rho \theta, \xi_{x}\right)\right|+\frac{1}{2}\left|\left(\rho^{2}, \xi_{x}\right)\right| \leq C\left(h^{2}\|\xi\|_{1}+h^{2}\|\theta\|\|\xi\|_{1}\right), \\
\left|\left(\rho_{x}, \xi\right)\right| \leq\left|\left(u \sigma, \xi_{x}\right)\right|+\frac{1}{2}\left|\left(u_{x} \xi, \xi\right)\right|+\left|\left(\sigma \xi, \xi_{x}\right)\right|+\frac{1}{2}\left|\left(\sigma^{2}, \xi_{x}\right)\right| \leq C\left(h^{2}\|\xi\|_{1}+\|\xi\|_{1}^{2}\right), \leq C\left(h^{2}\|\theta\|+h^{2}\|\xi\|\right) .
\end{gathered}
$$

Hence, from (3.6) we conclude that for $0 \leq t \leq T$,

$\frac{d}{d t}\left(\|\theta\|^{2}+\|\xi\|_{1}^{2}\right) \leq C\left(h^{3 / 2}\|\theta\|+h^{2}\|\xi\|_{1}\right)+C\left(\|\theta\|^{2}+\|\xi\|_{1}^{2}\right) \leq C\left[h^{3}+\left(\|\theta\|^{2}+\|\xi\|_{1}^{2}\right)\right]$.

By Gronwall's Lemma we obtain

$$
\|\theta(t)\|^{2}+\|\xi(t)\|_{1}^{2} \leq C\left[h^{3}+\|\theta(0)\|^{2}+\|\xi(0)\|_{1}^{2}\right],
$$

from which, in view of (3.2), we get

$$
\|\theta\|+\|\xi\|_{1} \leq C h^{3 / 2}, \quad 0 \leq t \leq T,
$$

and the estimates in $(i)$ follow. In addition, from (3.7) and the approximation properties of $S_{h}^{2}$ and $S_{h, 0}^{2}$ one may easily derive the following $L^{2}$ estimates of $F, G$, and $H$, that we note for further reference

$$
\|F\| \leq C\left(\|\xi\|+h^{2}\right),\|G\| \leq C h^{2},\|H\| \leq C\left(\|\xi\|+h^{2}\right) .
$$

We proceed now to prove the optimal-order error estimates in (ii). Equation (3.4) may be written in the form

$$
\xi_{t}=R_{h} v,
$$

where $v$ is the solution of the problem $v-\frac{1}{3} v^{\prime \prime}=-(\theta+\rho)_{x}-\frac{1}{2}(\eta \theta)_{x}-\frac{1}{2}\left(G-\frac{1}{2} \theta^{2}+3 H\right)_{x}-\sigma_{t}, \quad x \in[0,1], \quad v(0)=v(1)=0$.

Considering the weak form of (3.10) in $H_{0}^{1}$, and (3.7) and (3.8) we see that

$$
\|v\|_{1} \leq C h^{3 / 2} \text {. }
$$


In order to derive a bound for $\|v\|$, let $\zeta \in L^{2}$ and $V$ be the solution of the problem

$$
V-\frac{1}{3} V^{\prime \prime}=\zeta, \quad x \in[0,1], V(0)=V(1)=0 .
$$

Then, by (3.10)

$$
(v, \zeta)=a(v, V)=\left(\theta+\rho, V^{\prime}\right)+\frac{1}{2}\left(\eta \theta, V^{\prime}\right)+\frac{1}{2}\left(G-\frac{1}{2} \theta^{2}+3 H, V^{\prime}\right)-\left(\sigma_{t}, V\right) .
$$

From (3.3) with $\phi=1$ we see that $\left(\theta_{t}+\rho_{t}, 1\right)=0,0 \leq t \leq T$. Hence, $\int_{0}^{1}(\theta+$ $\rho) d x=\int_{0}^{1} \rho(x, 0) d x=: J=$ const. Therefore, if for $(x, t) \in[0,1] \times[0, T]$,

$$
\gamma(x, t):=\int_{0}^{x}(\theta(s, t)+\rho(s, t)) d s-x J
$$

it follows that $\gamma \in H_{0}^{1}$ and $\gamma_{x}=\theta+\rho-J$. Hence, (3.13) yields

$$
\begin{aligned}
(v, \zeta) & =\left(\gamma_{x}, V^{\prime}\right)+\frac{1}{2}\left(\eta \gamma_{x}, V^{\prime}\right)+\frac{1}{2}\left(G-\frac{1}{2} \theta^{2}+3 H-\eta \rho+\eta J, V^{\prime}\right)-\left(\sigma_{t}, V\right) \\
& =-\left(\gamma, V^{\prime \prime}\right)-\frac{1}{2}\left(\gamma, \eta V^{\prime \prime}+\eta_{x} V^{\prime}\right)+\frac{1}{2}\left(G-\frac{1}{2} \theta^{2}+3 H-\eta \rho+\eta J, V^{\prime}\right)-\left(\sigma_{t}, V\right) .
\end{aligned}
$$

Now, using (3.7), (3.8), the approximation and inverse properties of $S_{h}^{2}, S_{h, 0}^{2}$, and elliptic regularity in (3.12) we obtain $|(v, \zeta)| \leq\|\gamma\|\left\|V^{\prime \prime}\right\|+C\|\gamma\|\left(\left\|V^{\prime \prime}\right\|+\left\|V^{\prime}\right\|\right)$ $+C\left(h^{2}+\|\xi\|\right)\left\|V^{\prime}\right\|+C h^{2}\|V\| \leq C\left(h^{2}+\|\gamma\|+\|\xi\|\right)\|\zeta\|$, and conclude that

$$
\|v\| \leq C\left(h^{2}+\|\gamma\|+\|\xi\|\right) \text {. }
$$

Now let $W$ be the solution of the problem

$$
W-\frac{1}{3} W^{\prime \prime}=\xi, \quad x \in[0,1], W(0)=W(1)=0 .
$$

Using (3.9), (2.15), elliptic regularity in (3.16), and the estimates (3.11) and (3.15) gives $\left(\xi, \xi_{t}\right)=a\left(W, \xi_{t}\right)=a\left(W, R_{h} v\right)=a\left(v, R_{h} W\right)=a\left(v, R_{h} W-W\right)+a(W, v)=$ $a\left(v, R_{h} W-W\right)+(\xi, v) \leq C h\|\xi\|\|v\|_{1}+\|\xi\|\|v\| \leq C\left(h^{2}+\|\gamma\|+\|\xi\|\right)\|\xi\|$, from which it follows that

$$
\frac{1}{2} \frac{d}{d t}\|\xi\|^{2} \leq C\left(h^{4}+\|\gamma\|^{2}+\|\xi\|^{2}\right), \quad 0 \leq t \leq T .
$$

In order to obtain the required optimal-order estimate for $\|\xi\|$ from (3.17) we need a similar estimate for a suitable approximation of $\gamma$. For this purpose, observe that (3.3) yields, for $0 \leq t \leq T$ and $\phi \in S_{h}^{2}$,

$$
\left(\gamma_{x t}, \phi\right)+\frac{1}{2}\left((u \theta)_{x}, \phi\right)=-\left(w_{x}, \phi\right),
$$

where $w=\xi+\left(1+\frac{1}{2} \eta\right) \sigma-\frac{1}{2} \theta \xi+\frac{1}{2} F$; note that $w \in H_{0}^{1}$ and that (3.7) and (3.8) give $\|w\| \leq C\left(\|\xi\|+h^{2}\right)$. Using integration by parts and the definition of $\gamma$ in (3.18) yields, for $0 \leq t \leq T$,

$$
\left(\gamma_{t}, \phi^{\prime}\right)+\frac{1}{2}\left(u \gamma_{x}, \phi^{\prime}\right)=-\left(w-\frac{1}{2} u \rho+\frac{1}{2} u J, \phi^{\prime}\right) \quad \forall \phi \in S_{h}^{2} .
$$

Consider now the space $S_{h}^{-1}$ of discontinuous, piecewise constant functions relative to the partition $\left\{x_{j}\right\}$. Given any $\psi \in S_{h}^{-1}$ consider in (3.19) $\phi \in S_{h}^{2}$ such that $\phi^{\prime}=\psi$. Hence we have for $0 \leq t \leq T\left(\gamma_{t}, \psi\right)+\frac{1}{2}\left(u \gamma_{x}, \psi\right)=-(K, \psi) \forall \psi \in S_{h}^{-1}$, where $K:=w-\frac{1}{2} u \rho+\frac{1}{2} u J$ satisfies $\|K\| \leq C\left(\|\xi\|+h^{2}\right)$. Now taking $\psi=P_{0} \gamma$ in the above, where $P_{0}$ is the $L^{2}$-projection operator onto $S_{h}^{-1}$, yields, for $0 \leq t \leq T$,

$$
\frac{1}{2} \frac{d}{d t}\left\|P_{0} \gamma\right\|^{2}+\frac{1}{2}\left(u \gamma_{x}, P_{0} \gamma\right)=-\left(K, P_{0} \gamma\right) .
$$

Since $\left\|P_{0} \gamma-\gamma\right\| \leq C h\|\gamma\|_{1}$ (cf. e.g. (16.24) of [27]) and $\|\gamma\|_{1} \leq C h^{3 / 2}$ by (3.7), we have $\left|\left(u \gamma_{x}, P_{0} \gamma\right)\right|=\left|\left(u \gamma_{x}, P_{0} \gamma-\gamma\right)-\frac{1}{2}\left(u_{x} \gamma, \gamma\right)\right| \leq C\left(h\|\gamma\|_{1}^{2}+\|\gamma\|^{2}\right) \leq C\left(h\|\gamma\|_{1}^{2}\right.$ 
$\left.+\left\|P_{0} \gamma-\gamma\right\|^{2}+\left\|P_{0} \gamma\right\|^{2}\right) \leq C\left(h^{4}+\left\|P_{0} \gamma\right\|^{2}\right)$. Hence, (3.20) yields, for $0 \leq t \leq T$, that

$$
\frac{1}{2} \frac{d}{d t}\left\|P_{0} \gamma\right\|^{2} \leq C\left(h^{4}+\left\|P_{0} \gamma\right\|^{2}+\|\xi\|^{2}\right) .
$$

Now, from (3.17), since $\|\gamma\| \leq\left\|P_{0} \gamma-\gamma\right\|+\left\|P_{0} \gamma\right\| \leq C h^{5 / 2}+\left\|P_{0} \gamma\right\|$, we get for $0 \leq t \leq T$,

$$
\frac{1}{2} \frac{d}{d t}\|\xi\|^{2} \leq C\left(h^{4}+\left\|P_{0} \gamma\right\|^{2}+\|\xi\|^{2}\right) .
$$

Adding (3.21) and (3.22) we finally obtain by Gronwall's Lemma and (3.5) that

$$
\left\|P_{0} \gamma\right\|^{2}+\|\xi\|^{2} \leq C h^{4}, \quad 0 \leq t \leq T .
$$

Therefore, the first inequality of the conclusion $(i i)$ of the theorem holds; in addition, by similar estimates with the ones used above, we also obtain

$$
\|\gamma\| \leq C h^{2}, \quad 0 \leq t \leq T .
$$

Finally, we prove the second estimate of $(i i)$. Let $Z$ be the solution of the problem $Z-\frac{1}{3} Z^{\prime \prime}=\xi_{t}, x \in[0,1], Z(0)=Z(1)=0$. Then, by (3.9) $\left\|\xi_{t}\right\|^{2}=a\left(Z, \xi_{t}\right)=$ $a\left(Z, R_{h} v\right)=a\left(R_{h} Z, v\right)=a\left(R_{h} Z-Z, v\right)+\left(\xi_{t}, v\right)$. Hence, elliptic regularity and (3.11), (3.15), (3.23), (3.24) give

$$
\left\|\xi_{t}\right\|^{2} \leq C h\|Z\|_{2}\|v\|_{1}+\left\|\xi_{t}\right\|\|v\| \leq C h^{2}\left\|\xi_{t}\right\|
$$

i.e., $\left\|\xi_{t}\right\| \leq C h^{2}$, and the second estimate of (ii) follows.

Remark 3.1. It is not hard to see that the conclusions of Theorem 3.1 hold if $\eta_{h}(0)$ is any approximation of $\eta_{0}$ in $S_{h}^{2}$ with optimal-order $L^{2}$ rate of convergence. However, $u_{h}(0)$ has to be taken as $I_{h, 0} u_{0}$ or $R_{h} u_{0}$.

Remark 3.2. The superaccuracy estimate $\|\xi\|_{1}=O\left(h^{3 / 2}\right)$ of (3.7) combined with (2.3) and Sobolev's inequality yields the $L^{\infty}$ estimate $\left\|u-u_{h}\right\|_{\infty}=O\left(h^{3 / 2}\right)$ for $u$.

Remark 3.3. In the case of a quasiuniform mesh with $h=\max _{i}\left(x_{i+1}-x_{i}\right)$, one may easily check that the analog of Lemma 3.1 still holds; however, in the proof of Lemma 3.2 there is no longer cancellation from adjacent intervals and the conclusion is just that $\|\psi\| \leq C h$. As a consequence, the techniques of the proof of Theorem 3.1 now yield that $\left\|\eta-\eta_{h}\right\|=O(h)$ and $\left\|u-u_{h}\right\|_{1}=O(h)$, and, instead of the optimal-order estimates in (ii), that $\left\|u-u_{h}\right\|=O\left(h^{3 / 2}\right)$ and $\left\|u_{t}-u_{h t}\right\|=O\left(h^{3 / 2}\right)$. However, for the linearized problem

$$
\begin{aligned}
& \eta_{t}+u_{x}=0, \\
& u_{t}+\eta_{x}-\frac{1}{3} u_{x x t}=0, \quad(x, t) \in[0,1] \times[0, T], \\
& u(0, t)=0, \quad u(1, t)=0, \quad t \in[0, T], \\
& \eta(x, 0)=\eta_{0}(x), \quad u(x, 0)=u_{0}(x), \quad x \in[0,1],
\end{aligned}
$$

the last two estimates may be improved to yield optimal order, i.e., to give $\| u-$ $u_{h}\left\|=O\left(h^{2}\right),\right\| u_{t}-u_{h t} \|=O\left(h^{2}\right)$. The numerical experiments of section 6 suggest that $\left\|u-u_{h}\right\|=O\left(h^{2}\right)$ even in the nonlinear case, but we have not been able to prove this. 


\section{Semidiscretization With CUbiC Splines on a Uniform MeSh}

We consider again the uniform mesh on $[0,1]$ given by $x_{i}=(i-1) h, i=$ $1,2, \ldots, N+1$, where $N \geq 2$ is an integer and $h=1 / N$, and let $S_{h}^{4}:=\left\{\phi \in C^{2}\right.$ $\left.:\left.\phi\right|_{\left[x_{j}, x_{j+1}\right]} \in \mathbb{P}_{3}, 1 \leq j \leq N\right\}, S_{h, 0}^{4}=\left\{\phi \in S_{h}^{4}: \phi(0)=\phi(1)=0\right\}$, be the space of (the $C^{2}$ ) cubic splines on $[0,1]$ relative to the partition $\left\{x_{j}\right\}$, and the space of cubic splines that vanish at $x=0$ and at $x=1$. In this section we shall denote by $I_{h}: C^{1} \rightarrow S_{h}^{4}$ the interpolation operator, with the properties that for any $v \in C^{1}$, $\left(I_{h} v\right)\left(x_{i}\right)=v\left(x_{i}\right), 1 \leq i \leq N+1,\left(I_{h} v\right)^{\prime}\left(x_{k}\right)=v^{\prime}\left(x_{k}\right), k=1, N+1$, and let $I_{h, 0}: C_{0}^{1} \rightarrow S_{h, 0}^{4}$ be the analogous interpolant onto $S_{h, 0}^{4}$. It is well known that

$$
\sum_{j=0}^{2} h^{j}\left\|w-I_{h} w\right\|_{j} \leq C h^{k}\left\|w^{(k)}\right\|
$$

holds for any $w \in H^{k}$ for $k=2,3,4$ and that a similar estimate holds for $I_{h, 0} w$ if $w \in H^{k} \cap H_{0}^{1}$. More generally, [26], if $1 \leq k \leq 4$ and $0 \leq j<k$ we have that

$$
\min _{\chi \in S_{h}^{4}}\left\|(w-\chi)^{(j)}\right\| \leq C h^{k-j}\left\|w^{(k)}\right\| \quad \text { if } \quad w \in H^{k}
$$

and

$$
\min _{\chi \in S_{h}^{4}}\left\|(w-\chi)^{(j)}\right\|_{\infty} \leq C h^{k-j}\left\|w^{(k)}\right\|_{\infty} \quad \text { if } \quad w \in C^{k},
$$

and that a similar estimate holds in $S_{h, 0}^{4}$ for $w$ in $H^{k}$ or in $C^{k}$ that also vanishes at $x=0$ and $x=1$. (Of course, (4.1)-(4.3) hold for quasiuniform meshes as well.) We shall also use the notation and properties of the elliptic projection operator $R_{h}: H^{1} \rightarrow S_{h, 0}^{4}$ and the $L^{2}$ projection $P: L^{2} \rightarrow S_{h}^{4}$ introduced in section 2 for $r=4$, as well as the more general inverse inequalities

$\|\chi\|_{\beta} \leq C h^{-(\beta-\alpha)}\|\chi\|_{\alpha}, \quad 0 \leq \alpha \leq \beta \leq 2,\|\chi\|_{s, \infty} \leq C h^{-(s+1 / 2)}\|\chi\|, \quad 0 \leq s \leq 2$, valid for any $\chi \in S_{h}^{4}$ (or any $\chi \in S_{h, 0}^{4}$ ). As a consequence of these approximations and inverse properties it follows that $P$ is stable in $L^{\infty}$ and in $H^{1}$, and that $R_{h}$ is stable in $H_{0}^{1}$ and in $H^{2} \cap H_{0}^{1}$.

In this section, we let the standard Galerkin semidiscretization on $S_{h}^{4}$ of (SCB) be defined as follows: We seek $\eta_{h}:[0, T] \rightarrow S_{h}^{4}, u_{h}:[0, T] \rightarrow S_{h, 0}^{4}$, such that for $t \in[0, T]$,

$$
\begin{aligned}
& \left(\eta_{h t}, \phi\right)+\left(u_{h x}, \phi\right)+\frac{1}{2}\left(\left(\eta_{h} u_{h}\right)_{x}, \phi\right)=0 \quad \forall \phi \in S_{h}^{4}, \\
& a\left(u_{h t}, \chi\right)+\left(\eta_{h x}, \chi\right)+\frac{3}{2}\left(u_{h} u_{h x}, \chi\right)+\frac{1}{2}\left(\eta_{h} \eta_{h x}, \phi\right)=0 \quad \forall \chi \in S_{h, 0}^{4},
\end{aligned}
$$

with

$$
\eta_{h}(0)=I_{h} \eta_{0}, \quad u_{h}(0)=R_{h} u_{0} .
$$

The analogous semidiscretization of (CB) is defined similarly. We will denote by $\left\{\phi_{j}\right\}_{j=1}^{N+3}$ the usual $B$-spline basis of $S_{h}^{4}$ defined by the restrictions on $[0,1]$ of the functions $\phi_{j}(x)=\Phi\left(\frac{x}{h}-(j-2)\right)$, where $\Phi$ is the cubic spline on $\mathbb{R}$ with respect to the partition $\{-2,-1,0,1,2\}$ with support $[-2,2]$ and nodal values $\Phi(0)=1$, $\Phi( \pm 1)=1 / 4, \Phi( \pm 2)=0$. Thus, e.g., $\operatorname{supp}\left(\phi_{j}\right)=\left[x_{j-3}, x_{j+1}\right]$ and $\phi_{j}\left(x_{j-1}\right)=1$ for $4 \leq j \leq N$, etc. Before proving our main error estimate, we shall state and prove a series of auxiliary results. Our first lemma is a well-known result, the cubic spline analog of Lemma 3.1. 
Lemma 4.1. (i) Let $G_{i j}=\left(\phi_{j}, \phi_{i}\right), 1 \leq i, j \leq N+3$. Then, there exist positive constants $c_{1}$ and $c_{2}$ such that $c_{1} h|\gamma|^{2} \leq\langle G \gamma, \gamma\rangle \leq c_{2} h|\gamma|^{2} \forall \gamma \in \mathbb{R}^{N+3}$.

(ii) Let $b \in \mathbb{R}^{N+3}, \gamma=G^{-1} b$, and $\psi=\sum_{j=1}^{N+3} \gamma_{j} \psi_{j}$. Then $\|\psi\| \leq\left(c_{1} h\right)^{-1 / 2}|b|$.

(iii) Let $w \in C^{5}$. Then, there exists a constant $C_{1}=C_{1}\left(\left\|w^{(5)}\right\|_{\infty}\right)$ such that for any $\hat{x} \in\left[x_{i}, x_{i+1}\right]$,

$$
\left(w-I_{h} w\right)(x)=\frac{1}{4 !} w^{(4)}(\hat{x})\left(x-x_{i}\right)^{2}\left(x_{i+1}-x\right)^{2}+\tilde{g}(x), \quad x_{i} \leq x \leq x_{i+1},
$$

where $\|\tilde{g}\|_{\infty}+h\left\|\tilde{g}^{\prime}\right\|_{\infty} \leq C_{1} h^{5}$.

Proof. The proofs of $(i)$-(iii) are given in [18] in the case of periodic cubic splines. It is straightforward to adapt them to the case of $S_{h}^{4}$ and $I_{h}$ at hand.

We next prove a superaccuracy estimate for $P\left[(w \varepsilon)^{\prime}\right]$, where $\varepsilon$ is the error of the cubic spline interpolant of a sufficiently smooth function and $w$ is a $C^{1}$ weight. This estimate is a consequence of cancellation effects due to the uniform mesh and may be viewed as the cubic spline analog of Lemma 3.2.

Lemma 4.2. Let $v \in C^{5}$ and $w \in C^{1}$. If $\varepsilon=v-I_{h} v$ and $\psi \in S_{h}^{4}$ is such that $(\psi, \phi)=\left((w \varepsilon)^{\prime}, \phi\right) \forall \phi \in S_{h}^{4}$, then $\|\psi\| \leq C h^{3.5}$. If, in addition, $w(0)=w(1)=0$, then $\|\psi\| \leq C h^{4}$.

Proof. Let $b_{j}=\left((w \varepsilon)^{\prime}, \phi_{j}\right)=-\left(w \varepsilon, \phi_{j}^{\prime}\right), 1 \leq j \leq N+3$. In view of Lemma 4.1(ii) it suffices to show that $|b|=O\left(h^{4}\right)$. It is clear by (4.1) and the properties of the basis functions $\phi_{j}$ that $b_{j}=O\left(h^{4}\right)$ for all $j$. We will prove that actually $b_{j}=O\left(h^{5}\right)$ for $j=4,5, \ldots, N$, thus establishing that $|b|=O\left(h^{4}\right)$. Let $4 \leq j \leq N$. Using Lemma 4.1(iii) and putting $q(x)=x^{2}(h-x)^{2} / 4$ ! we have

$$
\begin{aligned}
\left(w \varepsilon, \phi_{j}^{\prime}\right)= & \sum_{k=0}^{3} \int_{x_{j-3+k}}^{x_{j-2+k}} w(x) \varepsilon(x) \phi_{j}^{\prime}(x) d x \\
= & v^{(4)}\left(x_{j-2}\right) \int_{x_{j-3}}^{x_{j-2}} w(x) q\left(x-x_{j-3}\right) \phi_{j}^{\prime}(x) d x \\
& +v^{(4)}\left(x_{j-1}\right) \int_{x_{j-2}}^{x_{j-1}} w(x) q\left(x-x_{j-2}\right) \phi_{j}^{\prime}(x) d x \\
& +v^{(4)}\left(x_{j-1}\right) \int_{x_{j-1}}^{x_{j}} w(x) q\left(x-x_{j-1}\right) \phi_{j}^{\prime}(x) d x \\
& +v^{(4)}\left(x_{j}\right) \int_{x_{j}}^{x_{j+1}} w(x) q\left(x-x_{j}\right) \phi_{j}^{\prime}(x) d x+O\left(h^{5}\right) \\
= & : v^{(4)}\left(x_{j-2}\right) J_{1}+v^{(4)}\left(x_{j-1}\right)\left(J_{2}+J_{3}\right)+v^{(4)}\left(x_{j}\right) J_{4}+O\left(h^{5}\right) .
\end{aligned}
$$

Hence, by Taylor's theorem we obtain

$$
\left(w \varepsilon, \phi_{j}^{\prime}\right)=v^{(4)}\left(x_{j-1}\right)\left(J_{1}+J_{2}+J_{3}+J_{4}\right)+O\left(h^{5}\right) .
$$

Suitable changes of variable in each of the four integrals yield $J_{1}=\int_{0}^{h} w(x+$ $\left.x_{j-3}\right) q(x) \phi_{4}^{\prime}(x) d x, J_{2}=\int_{0}^{h} w\left(x+x_{j-2}\right) q(x) \phi_{3}^{\prime}(x) d x, J_{3}=\int_{0}^{h} w\left(x+x_{j-1}\right) q(x) \phi_{2}^{\prime}(x)$ $d x, J_{4}=\int_{0}^{h} w\left(x+x_{j}\right) q(x) \phi_{1}^{\prime}(x) d x$. One-term Taylor expansions now give, for $x_{j \mu}=\left(x_{j-2}+x_{j-1}\right) / 2$, that $J_{1}+J_{2}+J_{3}+J_{4}=w\left(x_{j \mu}\right) \int_{0}^{h} q(x)\left[\phi_{1}^{\prime}(x)+\right.$ $\left.\phi_{2}^{\prime}(x)+\phi_{3}^{\prime}(x)+\phi_{4}^{\prime}(x)\right] d x+O\left(h^{5}\right)$. The last integral is equal to zero, since $\phi_{4}(x)=\phi_{1}(h-x), \phi_{3}(x)=\phi_{4}(h-x)$, and $q(x)=q(h-x)$ for $x \in[0, h]$. Hence 
$J_{1}+J_{2}+J_{3}+J_{4}=O\left(h^{5}\right)$, and (4.7) implies that $b_{j}=O\left(h^{5}\right)$. Thus, the first assertion of the lemma is verified. To prove the second assertion, suppose that $w(0)=0$. Then $b_{j}=O\left(h^{5}\right)$ for $j=1,2,3$. Indeed, using Lemma 4.1(iii), we have $-b_{1}=\left(w \varepsilon, \phi_{1}^{\prime}\right)=v^{(4)}(h) \int_{0}^{h} w(x) q(x) \phi_{1}^{\prime}(x) d x+O\left(h^{5}\right)=O\left(h^{5}\right)$. Similarly (cf. [6]), we may prove that $b_{2}=O\left(h^{5}\right)$ and $b_{3}=O\left(h^{5}\right)$. In addition, if $w(1)=0$, we have $b_{j}=O\left(h^{5}\right)$ for $j=N+1, N+2, N+3$. Hence, if $w$ vanishes at $x=0$ and $x=1,|b|=O\left(h^{5}\right)$ and the second assertion of the lemma follows by Lemma 4.1(ii).

We shall also derive a superaccuracy estimate for $P\left[(w e)^{\prime}\right]$, where $e$ is the error of the elliptic projection of a function $v \in C_{0}^{5}$ and $w$ is a $C^{1}$ weight. For this purpose, we first state two superconvergence results that follow from the analysis of Wahlbin in [28, and which are valid for interior nodes whose distance from the endpoints of the interval is at least of $O(h \ln 1 / h)$.

Proposition 4.1. Suppose that $v \in C_{0}^{5}$ and let $v_{h}=R_{h} v$ be its elliptic projection onto $S_{h, 0}^{4}$. Then the following hold:

(i) There exists a constant $C$ independent of $h$ such that

$$
\left|\left(v-v_{h}\right)^{\prime}\left(x_{i}\right)\right| \leq C h^{4}\|v\|_{W_{\infty}^{5}} \quad \text { provided } \quad \operatorname{dist}\left(x_{i}, \partial I\right) \geq C_{1} h \ln \frac{1}{h},
$$

where $C_{1}$ is a sufficiently large constant independent of $h$.

(ii) If $x_{i}, x_{i+1}$ are two adjacent nodes for which the second inequality in (4.8) holds and $e(x)=v(x)-v_{h}(x)$, we have

$$
e\left(x_{i+1}\right)-e\left(x_{i}\right)=O\left(h^{5}\right)\|v\|_{W_{\infty}^{5}} .
$$

Proof. (i) The estimate (4.8) follows from Corollary 1.6.2 of [28] (which is strictly valid when the elliptic projection is defined by $\left.\left(\tilde{v}_{h}^{\prime}, \phi^{\prime}\right)=\left(v^{\prime}, \phi^{\prime}\right) \forall \phi \in S_{h, 0}^{4}\right)$, combined with Theorem 1.3.1 of [28] which allows us to state the result for $v_{h}=R_{h} v$ defined as in section 2 of the paper at hand.

(ii) The cancellation property expressed by (4.9) is a consequence of the fact that $\tilde{e}(x)=v(x)-\tilde{v}_{h}(x)$ may be represented in the form

$$
\tilde{e}(x)=Q_{i}(x)+O\left(h^{5}\right)\|w\|_{W_{\infty}^{5}\left(x_{i}, x_{i+1}\right)}, x \in\left[x_{i}, x_{i+1}\right],
$$

where $Q_{i}(x)$ is a polynomial of degree four such that $Q_{i}\left(x_{i}\right)=Q_{i}\left(x_{i+1}\right)$. This representation follows from the remarks in Example 1.8.2 of [28] and by adapting the arguments of the proof in Section 1.8 of [28] (which are valid for $C^{1}$ Hermite cubics) to the case of the $C^{2}$ cubic splines at hand. Hence $\tilde{e}\left(x_{i+1}\right)-\tilde{e}\left(x_{i}\right)=$ $O\left(h^{5}\right)\|w\|_{W_{\infty}^{5}\left(x_{i}, x_{i+1}\right)}$, and (4.9) follows by the function values superconvergence estimate for elliptic projections given in Theorem 1.3.2 of [28].

In the next lemma we present some further formulas for the error $v-R_{h} v$ that will be used in the sequel.

Lemma 4.3. (i) Let $v \in C_{0}^{5}, v_{h}=R_{h} v, e=v-v_{h}$, and $1 \leq i \leq N$. Then, there exists a constant $C=C\left(\|w\|_{W_{\infty}^{5}}\right)$ such that for any $\hat{x} \in\left[x_{i}, x_{i+1}\right]$,

$$
e(x)=\gamma_{i}(x)+\frac{1}{4 !} v^{(4)}(\hat{x})\left(x-x_{i}\right)^{2}\left(x_{i+1}-x\right)^{2}+\delta_{i}(x), \quad x \in\left[x_{i}, x_{i+1}\right],
$$

where $\left\|\delta_{i}\right\|_{\infty} \leq C h^{5}$ and $\gamma_{i}$ is the cubic Hermite polynomial interpolating the values of $e$ and its derivative at the nodes $x_{i}$ and $x_{i+1}$. 
(ii) In addition to the hypotheses in (i), suppose that $x_{i}$ and $x_{i+1}$ satisfy the second inequality in (4.8). Then, there is a constant $C=C\left(\|v\|_{W_{\infty}^{5}}\right)$ such that for any $\hat{x} \in\left[x_{i}, x_{i+1}\right]$,

$$
e(x)=e\left(x_{i}\right)+\frac{1}{4 !} v^{(4)}(\hat{x})\left(x-x_{i}\right)^{2}\left(x_{i+1}-x\right)^{2}+\tilde{\delta}_{i}(x), \quad x \in\left[x_{i}, x_{i+1}\right],
$$

where $\left\|\tilde{\delta}_{i}\right\|_{\infty} \leq C h^{5}$.

Proof. (i) By the standard representation of the error of Hermite interpolation we have, since $v_{h} \in \mathbb{P}_{3}$ in $\left[x_{i}, x_{i+1}\right]$, that for $x \in\left[x_{i}, x_{i+1}\right]$ there holds $e(x)-\gamma_{i}=\frac{1}{4 !}$ $\left(x-x_{i}\right)^{2}\left(x_{i+1}-x\right)^{2} v^{(4)}\left(t_{x}\right)$ for some $t_{x} \in\left(x_{i}, x_{i+1}\right)$. Hence (4.10) follows from the mean-value theorem since $v \in C^{5}$.

(ii) We let $I=[0,1]$, and $K=\left\{x \in I: \operatorname{dist}(x, \partial I) \geq C_{1} h \ln 1 / h\right\}$. In view of (4.10) it suffices to show that $\gamma_{i}(x)=e\left(x_{i}\right)+O\left(h^{5}\right)\|v\|_{W_{\infty}^{5}}$ for $x_{i}, x_{i+1} \in K$. Now $\gamma_{i}(x)=e\left(x_{i}\right) A_{i, 1}(x)+e^{\prime}\left(x_{i}\right) B_{i, 1}(x)+e\left(x_{i+1}\right) A_{i, 2}(x)+e^{\prime}\left(x_{i+1}\right) B_{i, 2}(x)$, where

$$
\begin{array}{ll}
A_{i, 1}(x)=\left[1+\frac{2\left(x-x_{i}\right)}{h}\right] \frac{\left(x_{i+1}-x\right)^{2}}{h^{2}}, & B_{i, 1}(x)=\frac{\left(x-x_{i}\right)\left(x_{i+1}-x\right)^{2}}{h^{2}}, \\
A_{i, 2}(x)=\left[1+\frac{2\left(x_{i+1}-x\right)}{h}\right] \frac{\left(x-x_{i}\right)^{2}}{h^{2}}, & B_{i, 2}(x)=\frac{-\left(x_{i+1}-x\right)\left(x-x_{i}\right)^{2}}{h^{2}} .
\end{array}
$$

Hence, using (4.8) and (4.9) we see that $\gamma_{i}(x)=e\left(x_{i}\right) A_{i, 1}(x)+e\left(x_{i+1}\right) A_{i, 2}(x)+$ $O\left(h^{5}\right)\|v\|_{W_{\infty}^{5}}=e\left(x_{i}\right)+O\left(h^{5}\right)\|v\|_{W_{\infty}^{5}}$, which concludes the proof of the lemma.

We are now ready to prove the required estimate for $P\left[(w e)^{\prime}\right]$, where $w$ is a $C^{1}$ weight.

Lemma 4.4. Let $v \in C_{0}^{5}$ and $w \in C^{1}$. If $e=v-R_{h} v$ and $\psi \in S_{h}^{4}$ is such that $(\psi, \phi)=\left((w e)^{\prime}, \phi\right) \forall \phi \in S_{h}^{4}$, then $\|\psi\| \leq C h^{3.5} \sqrt{\ln 1 / h}$.

Proof. Let $b_{j}=\left((w e)^{\prime}, \phi_{j}\right), 1 \leq j \leq N+3$. In view of Lemma 4.1(ii) it suffices to show that $|b|=O\left(h^{4} \sqrt{\ln 1 / h}\right)$. It is clear by (2.2) and the properties of the basis functions $\phi_{j}$ that $b_{j}=O\left(h^{4}\right)$. We will show that for "most" of the the indices $j$ it is true that $b_{j}=O\left(h^{5}\right)$. Let $I=[0,1]$ and $K=\{x \in I: \operatorname{dist}(x, \partial I) \geq$ $\left.C_{1} h \ln 1 / h\right\}$. Since $x_{i}=(i-1) h, 1 \leq i \leq N+1$, it is clear that if $x_{i} \in K$, then $1+C_{1} \ln 1 / h \leq i \leq N+1-C_{1} \ln 1 / h$. Therefore $x_{i} \in K$ if and only if $i \in M=\left\{\nu \in \mathbb{N}: \nu \in\left[1+C_{1} \ln 1 / h, N+1-C_{1} \ln 1 / h\right]\right\}$. Let $\mu=\min M$ and $m=\max M$. We shall show that

$$
\left|b_{j}\right| \leq C h^{5}, \quad j=\mu+4, \ldots, m-1 .
$$

Indeed, for such $j$ we have

$$
\begin{aligned}
-b_{j}= & \left(w e, \phi_{j}^{\prime}\right)=\sum_{k=0}^{3} \int_{x_{j-3+k}}^{x_{j-2+k}} w(x) e(x) \phi_{j}^{\prime}(x) d x \\
= & \sum_{k=0}^{3}\left\{e\left(x_{j-3+k}\right) \int_{x_{j-3+k}}^{x_{j-2+k}} w(x) \phi_{j}^{\prime}(x) d x\right. \\
& \left.+\int_{x_{j-3+k}}^{x_{j-2+k}}\left(e(x)-e\left(x_{j-3+k}\right)\right) w(x) \phi_{j}^{\prime}(x) d x\right\} .
\end{aligned}
$$

Now using Lemma 4.3(ii) in each one of the second group of integrals of this expression and similar considerations as in the proof of Lemma 4.2, we see that

$$
-b_{j}=e\left(x_{j-3}\right) J_{1}+e\left(x_{j-2}\right) J_{2}+e\left(x_{j-1}\right) J_{3}+e\left(x_{j}\right) J_{4}+O\left(h^{5}\right),
$$


where $J_{i}=\int_{x_{j-4+i}}^{x_{j-3+i}} w(x) \phi_{j}^{\prime}(x) d x, 1 \leq i \leq 4$. But since $e\left(x_{i+1}\right)=e\left(x_{i}\right)+O\left(h^{5}\right)$ when $x_{i}, x_{i+1} \in K$ (by Proposition 4.1(ii)), and taking into account that the $J_{i}$ are bounded independently of $h$ and that $J_{1}+J_{2}+J_{3}+J_{4}=O(h)$ (by similar considerations as in the proof of Lemma 4.2), we finally obtain (4.12). By the definitions of $\mu$ and $m$ we now have

$$
\begin{aligned}
|b|^{2} & =\sum_{j=1}^{\mu+3} b_{j}^{2}+\sum_{j=\mu+4}^{m-1} b_{j}^{2}+\sum_{j=m}^{N+3} b_{j}^{2} \leq C\left[(\mu+3) h^{8}+h^{9}+(N+4-m) h^{8}\right] \\
& \leq C\left[\left(C_{2}+C_{1} \ln \frac{1}{h}\right) h^{8}+h^{9}\right] \leq C_{3} h^{8} \ln \frac{1}{h},
\end{aligned}
$$

where $C_{3}=C_{3}\left(\|v\|_{W_{\infty}^{5}},\|w\|_{W_{\infty}^{1}}, C_{1}\right)$, thus concluding the proof of the lemma.

The main result of this section follows.

Theorem 4.1. Let $h=1 / N$ be sufficiently small. Suppose that the solutions of ( $\mathrm{CB}$ and $(\mathrm{SCB})$ are such that $\eta \in C\left(0, T ; C^{5}\right), \eta_{t} \in C\left(0, T ; C^{4}\right), u, u_{t} \in$ $C\left(0, T ; C_{0}^{5}\right)$. Then the semidiscrete problem (4.5), (4.6) (and the analogous problem for (CB)) have unique solutions $\left(\eta_{h}, u_{h}\right)$ for $0 \leq t \leq T$ that satisfy

(i) $\max _{0 \leq t \leq T}\left\|\eta(t)-\eta_{h}(t)\right\| \leq C h^{3.5} \sqrt{\ln \frac{1}{h}}, \quad \max _{0 \leq t \leq T}\left\|u(t)-u_{h}(t)\right\|_{1} \leq C h^{3}$,

(ii) $\max _{0 \leq t \leq T}\left\|u(t)-u_{h}(t)\right\| \leq C h^{4} \sqrt{\ln \frac{1}{h}}, \quad \max _{0 \leq t \leq T}\left\|u_{t}(t)-u_{h t}(t)\right\| \leq C h^{4} \sqrt{\ln \frac{1}{h}}$.

Proof. Again, we give the proof in the case of ( $(\mathrm{SCB})$, noting that the analogous proof for ( $\mathrm{CB}$ ) follows as in Proposition 2.1. The technique of proof is basically the same as the one used in Theorem 3.1 and here we shall give the details that are different. We define now $\rho:=\eta-I_{h} \eta, \theta:=I_{h} \eta-\eta_{h}, \sigma:=u-R_{h} u, \xi:=R_{h} u-u_{h}$, and, arguing as in the proof of Theorem 3.1, we have for $0 \leq t \leq T$,

$$
\begin{aligned}
& \frac{1}{2} \frac{d}{d t}\left(\|\theta\|^{2}+\|\xi\|_{1}^{2}\right)+\left(\left(\left(1+\frac{1}{2} \eta\right) \sigma\right)_{x}, \theta\right)+\frac{1}{2}\left((u \theta)_{x}, \theta\right)+\frac{1}{2}\left(F_{x}, \theta\right) \\
& \quad+\left(\rho_{x}, \xi\right)+\frac{1}{2}\left((\eta \theta)_{x}, \xi\right)+\frac{1}{2}\left(G_{x}, \xi\right)+\frac{3}{2}\left(H_{x}, \xi\right)=-\left(\rho_{t}, \theta\right),
\end{aligned}
$$

where $F:=\eta \xi+u \rho-\rho \sigma-\rho \xi-\theta \sigma, G:=\eta \rho-\rho \theta-\frac{1}{2} \rho^{2}, H:=u \sigma+u \xi-\sigma \xi-\frac{1}{2} \sigma^{2}-$ $\frac{1}{2} \xi^{2}$. Using the approximation properties of $S_{h}^{4}$ and $S_{h, 0}^{4}$, integration by parts, and Lemmas 4.2 and 4.4 we have

$$
\begin{array}{ll}
\left|\left(\left(\left(1+\frac{1}{2} \eta\right) \sigma\right)_{x}, \theta\right)\right| \leq C h^{3.5} \sqrt{\ln \frac{1}{h}}\|\theta\|, & \left|\left((u \theta)_{x}, \theta\right)\right| \leq C\|\theta\|^{2}, \\
\left|\left(F_{x}, \theta\right)\right| \leq C\left(\|\xi\|_{1}\|\theta\|+h^{4}\|\theta\|+\|\theta\|^{2}\right), & \left|\left(\rho_{x}, \xi\right)\right| \leq C h^{4}\|\xi\|_{1}, \\
\left|\left((\eta \theta)_{x}, \xi\right)\right| \leq C\|\xi\|_{1}\|\theta\|, & \left|\left(G_{x}, \xi\right)\right| \leq C\left(\|\xi\|_{1}\|\theta\|+h^{4}\|\xi\|_{1}\right), \\
\left|\left(H_{x}, \xi\right)\right| \leq C\left(\|\xi\|^{2}+h^{4}\|\xi\|_{1}\right), & \left|\left(\rho_{t}, \theta\right)\right| \leq C h^{4}\|\theta\| .
\end{array}
$$

Therefore, (4.13) gives for $0 \leq t \leq T \frac{d}{d t}\left(\|\theta\|^{2}+\|\xi\|_{1}^{2}\right) \leq C\left(h^{7} \ln \frac{1}{h}+\|\theta\|^{2}+\|\xi\|_{1}^{2}\right)$, from which, by Gronwall's Lemma and (4.6), we get

$$
\|\theta\|+\|\xi\|_{1} \leq C h^{3.5} \sqrt{\ln \frac{1}{h}},
$$

and the estimates $(i)$ follow. In order to prove the estimates $(i i)$, we first note that that $\|F\| \leq C\left(\|\xi\|+h^{4}\right),\|G\| \leq C h^{4},\|H\| \leq C\left(\|\xi\|+h^{4}\right)$. Noting that $\xi_{t}=R_{h} v$, where $v$ is the solution of the problem

$v-\frac{1}{3} v^{\prime \prime}=-(\theta+\rho)_{x}-\frac{1}{2}(\eta \theta)_{x}-\frac{1}{2}\left(G-\frac{1}{2} \theta^{2}+3 H\right)_{x}, \quad x \in[0,1], \quad v(0)=v(1)=0$, 
we see by (4.14) that $\|v\|_{1} \leq C h^{3.5} \sqrt{\ln 1 / h}$. Arguing as in the proof of Theorem 3.1 we have now $\|v\| \leq C\left(h^{4}+\|\gamma\|+\|\xi\|\right)$, where $\gamma=\int_{0}^{x}(\theta(s, t)+\rho(s, t)) d s-$ $x \int_{0}^{1} \rho(s, 0) d s$. Then, it follows that $\frac{1}{2} \frac{d}{d t}\|\xi\|^{2} \leq C\left(h^{8}+\left\|P_{0} \gamma\right\|^{2}+\|\xi\|^{2}\right)$, where $P_{0}$ is the $L^{2}$-projection operator onto $S_{h}^{3}$, the space of $C^{1}$ piecewise quadratic functions relative to the partition $\left\{x_{j}\right\}$. We also have, in view of (4.14) and the estimate $\left\|P_{0} \gamma-\gamma\right\| \leq C h\|\gamma\|_{1}$, that $\frac{1}{2} \frac{d}{d t}\left\|P_{0} \gamma\right\|^{2} \leq C\left(h^{8} \ln \frac{1}{h}+\left\|P_{0} \gamma\right\|^{2}+\|\xi\|^{2}\right)$. From the last two inequalities and Gronwall's Lemma we obtain $\left\|P_{0} \gamma\right\|+\|\xi\| \leq$ $C h^{4} \sqrt{\ln \frac{1}{h}}$, from which the first estimate of $(i i)$ follows. The second estimate also follows along the lines of the proof of Theorem 3.1.

Remark 4.1. The results of the theorem also hold if we take as $\eta_{h}(0)$ any other approximation of $\eta_{0}$ in $S_{h}^{4}$ of optimal order of accuracy in $L^{2}$.

Remark 4.2. The superaccuracy estimate $\|\xi\|_{1}=O\left(h^{3.5} \sqrt{\ln 1 / h}\right)$ of (4.14), combined with (3.3) and Sobolev's inequality yield the $L^{\infty}$ estimate $\left\|u-u_{h}\right\|_{\infty}=$ $O\left(h^{3.5} \sqrt{\ln 1 / h}\right)$.

\section{Fully DisCRETE SCHEMES}

In this section we turn to the study of some temporal discretizations of the ode systems represented by the standard Galerkin spatial discretizations of (CB) or (SCB), We shall confine ourselves to explicit time stepping schemes in order to avoid the more costly implicit methods that require solving nonlinear systems of equations at each time step. Of course, with explicit methods there arises the issue of stability of the fully discrete schemes. We will not be exhaustive in our analysis but we will study as examples three simple, well-known explicit RungeKutta temporal discretizations, namely the explicit Euler scheme, the improved Euler, and the classical, four-stage Runge-Kutta method, of orders of accuracy 1, 2 , and 4 , respectively. These schemes require, respectively, stability conditions of the type $k=O\left(h^{2}\right), k=O\left(h^{4 / 3}\right)$, and $k \leq \lambda_{0} h$ for $\lambda_{0}$ sufficiently small, where $k$ is the time step. The reader will find full convergence proofs in 6 . Here, we shall analyze in detail the improved Euler time-stepping and just state the error estimate results for the two other schemes.

5.1. The explicit Euler scheme. Let $M$ be a positive integer, $k=T / M$ denote the (uniform) time step, and put $t^{n}=n k, n=0,1, \ldots, M$. We consider for example, the standard Galerkin semidiscretizations with piecewise linear, continuous functions on a uniform spatial mesh on $[0,1]$ with $h=1 / N$, given by the initialvalue problems (2.7), (3.2) and (2.9), (3.2) (with $S_{h}=S_{h}^{2}, S_{h, 0}=S_{h, 0}^{2}$ ) in the case of the (CB) and the (SCB) systems, respectively. We discretize the systems in time with the explicit Euler scheme. Hence, we seek for $0 \leq n \leq M H_{h}^{n} \in S_{h}^{2}, U_{h}^{n} \in S_{h, 0}^{2}$, approximations of the solution $\eta\left(x, t^{n}\right), u\left(x, t^{n}\right)$ of the (SCB $)$ system, such that for $0 \leq n \leq M-1$,

$$
\begin{aligned}
& \left.\left(H_{h}^{n+1}-H_{h}^{n}, \phi\right)+k\left(U_{h x}^{n}, \phi\right)+\frac{k}{2}\left(\left(H_{h}^{n} U_{h}^{n}\right)_{x}\right), \phi\right)=0 \quad \forall \phi \in S_{h}^{2}, \\
& a\left(U_{h}^{n+1}-U_{h}^{n}, \chi\right)+k\left(H_{h x}^{n}, \chi\right)+\frac{3 k}{2}\left(U_{h}^{n} U_{h x}^{n}, \chi\right)+\frac{k}{2}\left(H_{h}^{n} H_{h x}^{n}, \chi\right)=0 \quad \forall \chi \in S_{h, 0}^{2},
\end{aligned}
$$

with

$$
H_{h}^{0}=I_{h} \eta_{0}, \quad U_{h}^{0}=I_{h, 0} u_{0} .
$$


The full discretization of the semidiscrete (CB) system (2.7), (3.2) is defined analogously. The following result is proved in 6 .

Proposition 5.1. Suppose that the solutions $(\eta, u)$ of $(\mathrm{SCB})$ and $(\mathrm{CB})$ are sufficiently smooth on $[0, T]$. Then, if $\mu=k / h^{2}$, there is a constant $C=C(\mu)$, which is an increasing continuous function of $\mu$, such that

$$
\max _{0 \leq n \leq M}\left\|H_{h}^{n}-\eta\left(t^{n}\right)\right\| \leq C\left(k+h^{3 / 2}\right), \quad \max _{0 \leq n \leq M}\left\|U_{h}^{n}-u\left(t^{n}\right)\right\|_{1} \leq C(k+h),
$$

where $\left(H_{h}^{n}, U_{h}^{n}\right)$ satisfy (5.1), (5.2) or the analogous fully discrete scheme for (CB), as the case may be.

The condition $k=O\left(h^{2}\right)$ also seems to be necessary in some sense. Numerical experiments, whose results appear in [6], indicate that the accuracy of computations degenerated when we took $k=h^{\alpha}$ with decreasing $\alpha<2$.

5.2. The improved Euler method. We next study in detail the temporal discretization of the initial-value problems (2.7), (3.2) and (2.9), (3.2) by the explicit, second-order accurate "improved Euler" scheme, that may be written in the case of the ode $y^{\prime}=f(t, y)$ in the two-step form $y^{n, 1}=y^{n}+\frac{k}{2} f\left(t^{n}, y^{n}\right), y^{n+1}=y^{n}$ $+k f\left(t^{n}+\frac{k}{2}, y^{n, 1}\right)$. We first introduce some notation in order to write the fully discrete schemes more compactly. Let $A: L^{2} \rightarrow S_{h, 0}^{2}$ be defined for $f \in L^{2}$ by

$$
a(A f, \chi)=(f, \chi) \quad \forall \chi \in S_{h, 0}^{2},
$$

i.e., as the discrete solution operator such that $w_{h}=A f$, where $w_{h}$ is the standard Galerkin approximation in $S_{h, 0}^{2}$ of the solution of the two-point bvp $-\frac{1}{3} w^{\prime \prime}+w=$ $f, 0 \leq x \leq 1, w(0)=w(1)=0$. From (5.3) wave immediately that

$$
\|A f\|_{1} \leq C\|f\|_{-1},
$$

where the $\|\cdot\|_{-1}$ norm is defined for $f \in L^{2}$ by $\|f\|_{-1}=\sup \left\{\frac{(f, g)}{\|g\|_{1}}: g \in H_{0}^{1}, g \neq 0\right\}$. With this notation in place and as before letting $P$ be the $L^{2}$ projection operator onto $S_{h}^{2}$, we may rewrite the improved Euler fully discrete scheme corresponding to the (SCB) system as follows: For $0 \leq n \leq M$ we seek $H_{h}^{n} \in S_{h}^{2}, U_{h}^{n} \in S_{h, 0}^{2}$, and for $0 \leq n \leq M-1 H_{h}^{n, 1} \in S_{h}^{2}, U_{h}^{n, 1} \in S_{h, 0}^{2}$ such that

$$
\begin{aligned}
& H_{h}^{n, 1}-H_{h}^{n}+\frac{k}{2} P U_{h x}^{n}+\frac{k}{4} P\left(H_{h}^{n} U_{h}^{n}\right)_{x}=0, \\
& U_{h}^{n, 1}-U_{h}^{n}+\frac{k}{2} A H_{h x}^{n}+\frac{3 k}{4} A\left(U_{h}^{n} U_{h x}^{n}\right)+\frac{k}{4} A\left(H_{h}^{n} H_{h x}^{n}\right)=0, \\
& H_{h}^{n+1}-H_{h}^{n}+k P U_{h x}^{n, 1}+\frac{k}{2} P\left(H_{h}^{n, 1} U_{h}^{n, 1}\right)_{x}=0, \\
& U_{h}^{n+1}-U_{h}^{n}+k A H_{h x}^{n, 1}+\frac{3 k}{2} A\left(U_{h}^{n, 1} U_{h x}^{n, 1}\right)+\frac{k}{2} A\left(H_{h}^{n, 1} H_{h x}^{n, 1}\right)=0,
\end{aligned}
$$

for $0 \leq n \leq M-1$, with $H_{h}^{0}=I_{h} \eta_{0}, U_{h}^{0}=I_{h, 0} u_{0}$. The fully discrete approximation for (CB) is defined analogously.

We will prove error estimates for the scheme (5.5) and its (CB) analog by comparing $H_{h}^{n}$ with $I_{h} \eta\left(t^{n}\right)$ and $U_{h}^{n}$ with $I_{h, 0} u\left(t^{n}\right)$. For this purpose, it is useful to establish the following estimates of the truncation errors of the interpolants. (In the sequel we shall analyze the approximation of the (SCB system; the analogous results for (CB) follow as in section 2. Frequently, we shall suppress the $x$ variable, denoting, e.g., $\eta(\cdot, t)$ by $\eta(t)$ etc.) 
Lemma 5.1. Suppose that the solution $(\eta, u)$ of $(\underline{\mathrm{SCB}})$ is sufficiently smooth in $[0, T]$. Let $H(t)=I_{h} \eta(t), U(t)=I_{h, 0} u(t)$, and define $\psi(t) \in S_{h}^{2}, \zeta(t) \in S_{h, 0}^{2}$ for $0 \leq t \leq T$ by

$$
\begin{aligned}
H_{t}+P U_{x}+\frac{1}{2} P(H U)_{x} & =\psi, \\
U_{t}+A H_{x}+\frac{3}{2} A\left(U U_{x}\right)+\frac{1}{2} A\left(H H_{x}\right) & =\zeta .
\end{aligned}
$$

Then

$$
\|\psi\| \leq C h^{3 / 2}, \quad\left\|\psi_{t}\right\| \leq C h^{3 / 2},\|\zeta\|_{1} \leq C h^{2},\left\|\zeta_{t}\right\|_{1} \leq C h^{2},
$$

hold for $0 \leq t \leq T$. An analogous result holds for (CB).

Proof. Subtracting the equations $P\left(\eta_{t}+u_{x}+\frac{1}{2}(u \eta)_{x}\right)=0$ and (5.6), and putting $\rho:=\eta-I_{h} \eta, \sigma:=u-I_{h, 0} u$, we obtain $P\left(\rho_{t}+\left[\left(1+\frac{1}{2} \eta\right) \sigma\right]_{x}+\frac{1}{2}(u \rho)_{x}-\frac{1}{2}(\rho \sigma)_{x}\right)$ $=-\psi$. Therefore, using the approximation properties of $S_{h}^{2}$ and $S_{h, 0}^{2}$, and Lemma 3.2 , we have

$\|\psi\| \leq\left\|\rho_{t}\right\|+\left\|P\left[\left(1+\frac{1}{2} \eta\right) \sigma\right]_{x}\right\|+\frac{1}{2}\left\|P(u \rho)_{x}\right\|+\left\|(\rho \sigma)_{x}\right\| \leq C\left(h^{2}+h^{3 / 2}+h^{2}+h^{3}\right) \leq C h^{3 / 2}$.

Similarly, since e.g. by Lemma $3.2\left\|P\left[\left(1+\frac{1}{2} \eta\right) \sigma\right]_{x t}\right\| \leq\left\|P\left(\frac{1}{2} \eta_{t} \sigma\right)_{x}\right\|+\| P[(1+$ $\left.\left.\frac{1}{2} \eta\right) \sigma_{t}\right]_{x} \| \leq C h^{3 / 2}$, we have

$$
\begin{aligned}
\left\|\psi_{t}\right\| & \leq\left\|\rho_{t}\right\|+\left\|P\left[\left(1+\frac{1}{2} \eta\right) \sigma\right]_{x t}\right\|+\frac{1}{2}\left\|P(u \rho)_{x t}\right\|+\left\|(\rho \sigma)_{x t}\right\| \\
& \leq C\left(h^{2}+h^{3 / 2}+h^{2}+h^{3}\right) \leq C h^{3 / 2} .
\end{aligned}
$$

Now, note that for any $\chi \in S_{h, 0}^{2},(5.3)$ and the fact that $\left(\sigma_{t}^{\prime}, \chi^{\prime}\right)=0$ yield

$$
\begin{aligned}
a\left(A\left(u_{t}-\frac{1}{3} u_{t x x}\right)-U_{t}, \chi\right) & =\left(u_{t}, \chi\right)+\frac{1}{3}\left(u_{t x}, \chi^{\prime}\right)-a\left(U_{t}, \chi\right) \\
=a\left(\sigma_{t}, \chi\right)=\left(\sigma_{t}, \chi\right) & =a\left(A \sigma_{t}, \chi\right) .
\end{aligned}
$$

Hence $A\left(u_{t}-\frac{1}{3} u_{t x x}\right)-U_{t}=A \sigma_{t}$, which implies, in view of the second pde of (SCB) that $A \sigma_{t}+U_{t}+A\left(\eta_{x}+\frac{3}{2} u u_{x}+\frac{1}{2} \eta \eta_{x}\right)=0$. Now subtracting this equation from (5.7) we see, after some algebra, that

$$
A\left(\sigma_{t}+\rho_{x}+\frac{3}{2}\left[(u \sigma)_{x}-\sigma \sigma_{x}\right]+\frac{1}{2}\left[(\eta \rho)_{x}-\rho \rho_{x}\right]\right)=-\zeta .
$$

Therefore, using (5.4) and the approximation properties of $S_{h}^{2}$ and $S_{h, 0}^{2}$, we obtain

$$
\begin{aligned}
\|\zeta\|_{1} & \leq C\left(\left\|\sigma_{t}\right\|_{-1}+\left\|\rho_{x}\right\|_{-1}+\left\|\left(u \sigma-\frac{1}{2} \sigma^{2}\right)_{x}\right\|_{-1}+\left\|\left(\eta \rho-\frac{1}{2} \rho^{2}\right)_{x}\right\|_{-1}\right) \\
& \leq C\left(\left\|\sigma_{t}\right\|+\|\rho\|+\|u \sigma\|+\left\|\sigma^{2}\right\|+\|\eta \rho\|+\left\|\rho^{2}\right\|\right) \leq C h^{2} .
\end{aligned}
$$

Similarly, after differentiating (5.9) with respect to $t$, we see that $\left\|\zeta_{t}\right\|_{1} \leq C h^{2}$, thus ending the proof. The same results hold for (CB) of course.

In order to study the consistency and convergence of the fully discrete schemes we let $H^{n}=H\left(t^{n}\right)=I_{h} \eta\left(t^{n}\right), U^{n}=U\left(t^{n}\right)=I_{h, 0} u\left(t^{n}\right)$, where $(\eta, u)$ is the solution of ( $\underline{\mathrm{SCB}})$, and define $\left(H^{n, 1}, U^{n, 1}\right) \in S_{h}^{2} \times S_{h, 0}^{2}$ for $0 \leq n \leq M-1$ by the equations

$$
\begin{aligned}
& H^{n, 1}-H^{n}+\frac{k}{2} P U_{x}^{n}+\frac{k}{4} P\left(H^{n} U^{n}\right)_{x}=0, \\
& U^{n, 1}-U^{n}+\frac{k}{2} A H_{x}^{n}+\frac{3 k}{2} A\left(U^{n} U_{x}^{n}\right)+\frac{k}{4} A\left(H^{n} H_{x}^{n}\right)=0 .
\end{aligned}
$$

In the case of ( $(\overline{\mathrm{CB}}), H^{n, 1}, U^{n, 1}$ are defined analogously. Our consistency result follows. 
Lemma 5.2. Suppose that the solution $(\eta, u)$ of $(\underline{\mathrm{SCB}})$ is sufficiently smooth and let $\lambda=k / h$. Define, for $0 \leq n \leq M-1, \delta_{1}^{n}, \delta_{2}^{n}$ by the equations

$$
\begin{aligned}
& \delta_{1}^{n}=H^{n+1}-H^{n}+k P U_{x}^{n, 1}+\frac{k}{2} P\left(H^{n, 1} U^{n, 1}\right)_{x}, \\
& \delta_{2}^{n}=U^{n+1}-U^{n}+k A H_{x}^{n, 1}+\frac{3 k}{2} A\left(U^{n, 1} U_{x}^{n, 1}\right)+\frac{k}{2} A\left(H^{n, 1} H_{x}^{n, 1}\right) .
\end{aligned}
$$

Then, there exists a constant $C_{1}=C_{1}(\lambda)$, which is a polynomial of $\lambda$ of degree one, such that

$$
\max _{0 \leq n \leq M-1}\left(\left\|\delta_{1}^{n}\right\|+\left\|\delta_{2}^{n}\right\|_{1}\right) \leq C_{1} k\left(k^{2}+h^{3 / 2}\right) .
$$

The analogous result holds for (CB) as well.

Proof. Let $0 \leq n \leq M-1$. By (5.10), (5.6) and (5.7) we have

$$
H^{n, 1}=H^{n}+\frac{k}{2} H_{t}^{n}-\frac{k}{2} \psi^{n}, \quad U^{n, 1}=U^{n}+\frac{k}{2} U_{t}^{n}-\frac{k}{2} \zeta^{n} .
$$

From these expressions, after some algebra, we obtain $H^{n, 1} U^{n, 1}=H^{n} U^{n}+\frac{k}{2}(H U)_{t}^{n}$ $+w_{1}^{n}$, where

$$
w_{1}^{n}:=\frac{k^{2}}{4} H_{t}^{n} U_{t}^{n}-\frac{k}{2}\left(U^{n}+\frac{k}{2} U_{t}^{n}\right) \psi^{n}-\frac{k}{2}\left(H^{n}+\frac{k}{2} H_{t}^{n}\right) \zeta^{n}+\frac{k^{2}}{4} \psi^{n} \zeta^{n} .
$$

Hence, by (5.11), (5.13), (5.8), and the above we obtain

$$
\delta_{1}^{n}=H^{n+1}-H^{n}-k H_{t}^{n}-\frac{k^{2}}{2} H_{t t}^{n}+k \psi^{n}+\frac{k^{2}}{2} \psi_{t}^{n}-\frac{k^{2}}{2} P \zeta_{x}^{n}+\frac{k}{2} P w_{1 x}^{n} .
$$

Now, (5.14), in view of (5.8) and the approximation and inverse properties of $S_{h}^{2}$ and $S_{h, 0}^{2}$, gives

$$
\begin{aligned}
\left\|w_{1}^{n}\right\|_{1} \leq & C\left(k^{2}\left\|H_{t}^{n}\right\|_{1}\left\|U_{t}^{n}\right\|_{1}+k\left\|\psi^{n}\right\|_{1}\left(\left\|U^{n}\right\|_{1}+k\left\|U_{t}^{n}\right\|_{1}\right)\right. \\
& \left.+k\left\|\zeta^{n}\right\|_{1}\left(\left\|H^{n}\right\|_{1}+k\left\|H_{t}^{n}\right\|_{1}\right)+k^{2}\left\|\psi^{n}\right\|_{1}\left\|\zeta^{n}\right\|_{1}\right) \\
\leq & c\left(k^{2}+k h^{-1} h^{3 / 2}(1+c k)+k h^{2}(1+c k)+k^{2} h^{-1} h^{7 / 2}\right) \leq c\left(k^{2}+\lambda h^{3 / 2}\right) .
\end{aligned}
$$

Therefore, by Taylor's theorem and (5.8) we have

$$
\left\|\delta_{1}^{n}\right\| \leq c\left(k^{3}+k h^{3 / 2}+k^{2} h^{3 / 2}+k^{2} h^{2}+k\left(k^{2}+\lambda h^{3 / 2}\right) \leq C_{1} k\left(k^{2}+h^{3 / 2}\right),\right.
$$

where $C_{1}$ is a constant that is a polynomial of $\lambda$ of degree one with positive coefficients. (Such constants will be generically denoted by $C_{1}$ in the sequel of this proof.) In order to estimate $\left\|\delta_{2}^{n}\right\|_{1}$ note that by (5.13),

$$
U^{n, 1} U_{x}^{n, 1}=U^{n} U_{x}^{n}+\frac{k}{2}\left(U U_{x}\right)_{t}^{n}+w_{2}^{n},
$$

where $w_{2}^{n}:=\frac{k^{2}}{4} U_{t}^{n} U_{t x}^{n}-\frac{k}{2}\left(\left(U^{n}+\frac{k}{2} U_{t}^{n}\right) \zeta^{n}\right)_{x}+\frac{k^{2}}{4} \zeta^{n} \zeta_{x}^{n}$. By (5.8) and the approximation properties of $S_{h, 0}^{2}$ we have

$$
\left\|w_{2}^{n}\right\| \leq C\left(k^{2}+k h^{2}\right) .
$$

Similarly,

$$
H^{n, 1} H_{x}^{n, 1}=H^{n} H_{x}^{n}+\frac{k}{2}\left(H H_{x}\right)_{t}^{n}+w_{3}^{n},
$$

where $w_{3}^{n}:=\frac{k^{2}}{4} H_{t}^{n} H_{t x}^{n}-\frac{k}{2}\left(\left(H^{n}+\frac{k}{2} H_{t}^{n}\right) \psi^{n}\right)_{x}+\frac{k^{2}}{4} \psi^{n} \psi_{x}^{n}$. By (5.8) and the approximation and inverse properties of $S_{h}^{2}$ we have

$$
\left\|w_{3}^{n}\right\| \leq C\left(k^{2}+\lambda h^{3 / 2}\right) .
$$

By (5.12), (5.14), (5.17), and (5.19), we see now that $\delta_{2}^{n}=\left(U^{n+1}-U^{n}-k U_{t}^{n}\right.$ $\left.-\frac{k^{2}}{2} U_{t t}^{n}\right)+k \zeta^{n}+\frac{k^{2}}{2} \zeta_{t}^{n}-\frac{k^{2}}{2} A \psi_{x}^{n}+\frac{3 k}{2} A w_{2}^{n}+\frac{k}{2} A w_{3}^{n}$. Therefore, by Taylor's theorem, (5.8), (5.4), (5.18), (5.20), $\left\|\delta_{2}^{n}\right\|_{1} \leq c\left(k^{3}+k h^{2}+k^{2} h^{2}+k^{2} h^{3 / 2}+k^{3}+\right.$ 
$\left.k^{2} h^{2}+k^{3}+\lambda k h^{3 / 2}\right) \leq C_{1} k\left(k^{2}+h^{3 / 2}\right)$, which, with (5.16), concludes the proof of the lemma. The case of ( $(\mathrm{CB})$ is entirely analogous.

For the stability and convergence of the fully discrete scheme it is sufficient to suppose that $k=O\left(h^{4 / 3}\right)$ as the following result shows.

Proposition 5.2. Suppose that the solutions $(\eta, u)$ of $(\underline{\mathrm{SCB}})$ and $(\mathrm{CB})$ are sufficiently smooth on $[0, T]$. Then, if $\mu=k / h^{4 / 3}$, there is a constant $C=C(\mu)$, which is an increasing continuous function of $\mu$, such that

$$
\max _{0 \leq n \leq M}\left\|H_{h}^{n}-\eta\left(t^{n}\right)\right\| \leq C\left(k^{2}+h^{3 / 2}\right), \quad \max _{0 \leq n \leq M}\left\|U_{h}^{n}-u\left(t^{n}\right)\right\|_{1} \leq C\left(k^{2}+h\right) .
$$

Proof. We consider (SCB), and put $\varepsilon^{n}=H^{n}-H_{h}^{n}, e^{n}=U^{n}-U_{h}^{n}, \theta^{n}=H^{n, 1}-H_{h}^{n, 1}$, and $\xi^{n}=U^{n, 1}-U_{h}^{n, 1}$. We will show that

$$
\max _{0 \leq n \leq M}\left(\left\|\varepsilon^{n}\right\|+\left\|e^{n}\right\|_{1}\right) \leq C\left(k^{2}+h^{3 / 2}\right),
$$

from which the conclusion of the proposition follows. From (5.6), (5.11), (5.12) we have, for $0 \leq n \leq M-1$,

$$
\varepsilon^{n+1}=\varepsilon^{n}-k P \xi_{x}^{n}-\frac{k}{2} P\left(H^{n, 1} U^{n, 1}-H_{h}^{n, 1} U_{h}^{n, 1}\right)_{x}+\delta_{1}^{n},
$$

and

$e^{n+1}=e^{n}-k A \theta_{x}^{n}-\frac{3 k}{2} A\left(U^{n, 1} U_{x}^{n, 1}-U_{h}^{n, 1} U_{h x}^{n, 1}\right)-\frac{k}{2} A\left(H^{n, 1} H_{x}^{n, 1}-H_{h}^{n, 1} H_{h x}^{n, 1}\right)+\delta_{2}^{n}$.

From Lemma 5.2 we have an estimate of $\left\|\delta_{1}^{n}\right\|+\left\|\delta_{2}^{n}\right\|_{1}$. Our goal is to obtain suitable estimates of the remaining terms of the right-hand sides of (5.22) and (5.23) in terms of $\left\|\varepsilon^{n}\right\|+\left\|e^{n}\right\|_{1}$. To do this, note first that (5.10) and (5.6) give $\theta^{n}=\varepsilon^{n}-\frac{k}{2} P e_{x}^{n}-$ $\frac{k}{4} P\left(H^{n} U^{n}-H_{h}^{n} U_{h}^{n}\right)_{x}$; but $H^{n} U^{n}-H_{h}^{n} U_{h}^{n}=H^{n} e^{n}-\varepsilon^{n} e^{n}+U^{n} \varepsilon^{n}$. Therefore,

$$
\theta^{n}=\varepsilon^{n}-\frac{k}{4} \rho_{1}^{n}-\frac{k}{2} \omega_{1}^{n},
$$

where

$$
\rho_{1}^{n}:=P\left(U^{n} \varepsilon^{n}\right)_{x}
$$

and

$$
\omega_{1}^{n}:=P e_{x}^{n}+\frac{1}{2} P\left(H^{n} e^{n}\right)_{x}-\frac{1}{2} P\left(\varepsilon^{n} e^{n}\right)_{x} .
$$

Similarly, $\xi^{n}=e^{n}-\frac{k}{2} A \varepsilon_{x}^{n}-\frac{3 k}{4} A\left(U^{n} U_{x}^{n}-U_{h}^{n} U_{h x}^{n}\right)-\frac{k}{4} A\left(H^{n} H_{x}^{n}-H_{h}^{n} H_{h x}^{n}\right)$; but $U^{n} U_{x}^{n}-U_{h} U_{h x}^{n}=\left(U^{n} e^{n}\right)_{x}-e^{n} e_{x}^{n}, H^{n} H_{x}^{n}-H_{h}^{n} H_{h x}^{n}=\left(H^{n} \varepsilon^{n}\right)_{x}-\varepsilon^{n} \varepsilon_{x}^{n}$. Therefore,

$$
\xi^{n}=e^{n}-\frac{k}{2} \omega_{2}^{n}
$$

where

$$
\omega_{2}^{n}:=A \varepsilon_{x}^{n}+\frac{3}{2} A\left(U^{n} e^{n}\right)_{x}-\frac{3}{2} A\left(e^{n} e_{x}^{n}\right)+\frac{1}{2} A\left(H^{n} \varepsilon^{n}\right)_{x}-\frac{1}{2} A\left(\varepsilon^{n} \varepsilon_{x}^{n}\right) .
$$

In addition, by (5.24) we have

$$
H^{n, 1} U^{n, 1}-H_{h}^{n, 1} U_{h}^{n, 1}=U^{n} \varepsilon^{n}-\frac{k}{4} U^{n} \rho_{1}^{n}-\frac{k}{2} U^{n} \omega_{1}^{n}+\omega_{3}^{n},
$$

where

$$
\omega_{3}^{n}:=\left(U^{n, 1}-U^{n}\right) \theta^{n}+H^{n, 1} \xi^{n}-\theta^{n} \xi^{n} .
$$

From (5.22), (5.24)-(5.29) we therefore conclude that for $0 \leq n \leq M-1$,

$$
\varepsilon^{n+1}=\varepsilon^{n}-\frac{k}{2} \rho_{1}^{n}+\frac{k^{2}}{8} \rho_{2}^{n}-k \omega_{4}^{n}+\delta_{1}^{n},
$$


where

$$
\rho_{2}^{n}:=P\left(U^{n} \rho_{1}^{n}\right)_{x}
$$

and

$$
\omega_{4}^{n}:=P e_{x}^{n}-\frac{k}{2} P \omega_{2 x}^{n}-\frac{k}{4} P\left(U^{n} \omega_{1}^{n}\right)_{x}+\frac{1}{2} P \omega_{3 x}^{n} .
$$

Finally, using the identities $U^{n, 1} U_{x}^{n, 1}-U_{h}^{n, 1} U_{h x}^{n, 1}=\left(U^{n, 1} \xi^{n}\right)_{x}-\xi^{n} \xi_{x}^{n}$ and $H^{n, 1} H_{x}^{n, 1}-$ $H_{h}^{n, 1} H_{h x}^{n, 1}=\left(H^{n, 1} \theta^{n}\right)_{x}-\theta^{n} \theta_{x}^{n}$, we obtain from (5.23) that for $0 \leq n \leq M-1$, (5.34) $e^{n+1}=e^{n}-k A \theta_{x}^{n}-\frac{3 k}{2} A\left(\left(U^{n, 1} \xi^{n}\right)_{x}-\xi^{n} \xi_{x}^{n}\right)-\frac{k}{2} A\left(\left(H^{n, 1} \theta^{n}\right)_{x}-\theta^{n} \theta_{x}^{n}\right)+\delta_{2}^{n}$.

We now estimate the various terms in the right-hand sides of (5.31) and (5.34). Let $0 \leq n^{*} \leq M-1$ be the maximal index for which

$$
\left\|\varepsilon^{n}\right\|_{1}+\left\|e^{n}\right\|_{1} \leq 1, \quad 0 \leq n \leq n^{*} .
$$

Then, by (5.26), the approximation properties of $S_{h}^{2}$ and (5.35), we have, for $0 \leq$ $n \leq n^{*}$,

$$
\left\|\omega_{1}^{n}\right\| \leq\left\|e^{n}\right\|_{1}+C\left\|H^{n}\right\|_{1}\left\|e^{n}\right\|_{1}+C\left\|\varepsilon^{n}\right\|_{1}\left\|e^{n}\right\|_{1} \leq C\left\|e^{n}\right\|_{1} .
$$

By (5.28), the approximation properties of $S_{h}^{2}, S_{h, 0}^{2}$, and (5.4) there follows for $0 \leq$ $n \leq n^{*}$,

$$
\left\|\omega_{2}^{n}\right\|_{1} \leq C\left(\left\|\varepsilon^{n}\right\|+\left\|e^{n}\right\|+\left\|e^{n}\right\|_{1}\left\|e^{n}\right\|+\left\|\varepsilon^{n}\right\|+\left\|\varepsilon^{n}\right\|_{1}\left\|\varepsilon^{n}\right\|\right) \leq C\left(\left\|\varepsilon^{n}\right\|+\left\|e^{n}\right\|\right) .
$$

Hence, by (5.27), for $0 \leq n \leq n^{*}$,

$$
\left\|\xi^{n}\right\|_{1} \leq C\left(\left\|\varepsilon^{n}\right\|+\left\|e^{n}\right\|_{1}\right) .
$$

In addition, by (5.24), (5.25), (5.36) and the inverse assumptions we have for $0 \leq$ $n \leq n^{*}$,

$\left\|\theta^{n}\right\| \leq\left\|\varepsilon^{n}\right\|+C \frac{k}{2}\left\|\varepsilon^{n}\right\|+C\left\|e^{n}\right\|_{1} \leq(1+C \lambda)\left\|\varepsilon^{n}\right\|+C\left\|e^{n}\right\|_{1} \leq C_{\lambda}\left(\left\|\varepsilon^{n}\right\|+\left\|e^{n}\right\|_{1}\right)$, where we have put $\lambda=k / h$; in what follows, $C_{\lambda}$ will denote various constants that depend polynomially on $\lambda$. Note also that in view of (5.35) we have for $0 \leq n \leq$ $n^{*}$, from (5.36), (5.25) and inverse inequalities,

(5.40) $\left\|\theta^{n}\right\|_{1} \leq\left\|\varepsilon^{n}\right\|_{1}+C k\left\|\rho_{1}^{n}\right\|_{1}+C k\left\|\omega_{1}^{n}\right\|_{1} \leq\left\|\varepsilon^{n}\right\|_{1}+C \lambda\left\|\rho_{1}^{n}\right\|+C \lambda\left\|\omega_{1}^{n}\right\| \leq C_{\lambda}$.

By (5.30), (5.13), (5.8), (5.39), (5.38) and (5.35) we have for $0 \leq n \leq n^{*}$,

$$
\begin{aligned}
\left\|\omega_{3}^{n}\right\| & \leq C\left(\left\|U^{n, 1}-U^{n}\right\|_{1}\left\|\theta^{n}\right\|+\left\|H^{n, 1}\right\|\left\|\xi^{n}\right\|_{1}+\left\|\theta^{n}\right\|\left\|\xi^{n}\right\|_{1}\right) \\
& \leq C\left(\left(k+h^{2}\right)\left\|\theta^{n}\right\|+\left\|\xi^{n}\right\|_{1}+\left\|\theta^{n}\right\|\left\|\xi^{n}\right\|_{1}\right) \\
& \leq C_{\lambda}\left(\left\|\varepsilon^{n}\right\|+\left\|e^{n}\right\|_{1}\right) .
\end{aligned}
$$

Also, by (5.39), (5.38), (5.40), for $0 \leq n \leq n^{*}$,

$$
\begin{aligned}
\left\|\omega_{3}^{n}\right\|_{1} & \leq C\left(\left\|U^{n, 1}-U^{n}\right\|_{1}\left\|\theta^{n}\right\|_{1}+\left\|H^{n, 1}\right\|_{1}\left\|\xi^{n}\right\|_{1}+\left\|\theta^{n}\right\|_{1}\left\|\xi^{n}\right\|_{1}\right) \\
& \leq\left(k+h^{2}\right) h^{-1} C_{\lambda}\left(\left\|\varepsilon^{n}\right\|+\left\|e^{n}\right\|_{1}\right)+C_{\lambda}\left(\left\|\varepsilon^{n}\right\|+\left\|e^{n}\right\|_{1}\right)+C_{\lambda}\left(\left\|\varepsilon^{n}\right\|+\left\|e^{n}\right\|_{1}\right) \\
& \leq C_{\lambda}\left(\left\|\varepsilon^{n}\right\|+\left\|e^{n}\right\|_{1}\right) .
\end{aligned}
$$

Hence, by (5.33), (5.37), (5.36), (5.41) and the inverse inequalities we have for $0 \leq n \leq n^{*}\left\|\omega_{4}^{n}\right\| \leq\left\|e^{n}\right\|_{1}+C k\left(\left\|\varepsilon^{n}\right\|+\left\|e^{n}\right\|\right)+C \lambda\left\|e^{n}\right\|_{1}+C_{\lambda}\left(\left\|\varepsilon^{n}\right\|+\left\|e^{n}\right\|_{1}\right) \leq$ 
$C_{\lambda}\left(\left\|\varepsilon^{n}\right\|+\left\|e^{n}\right\|_{1}\right)$. Therefore, in the right-hand side of (5.31) we have for $0 \leq n \leq$ $n^{*}$ in view of Lemma 5.2,

$$
\left\|-k \omega_{4}^{n}+\delta_{1}^{n}\right\| \leq C_{\lambda} k\left(\left\|\varepsilon^{n}\right\|+\left\|e^{n}\right\|_{1}\right)+C_{\lambda} k\left(k+h^{3 / 2}\right) .
$$

We embark now upon obtaining a sharp $L^{2}$-estimate of the remaining term $\varepsilon^{n}-$ $\frac{k}{2} \rho_{1}^{n}+\frac{k^{2}}{8} \rho_{2}^{n}$ in (5.31). We have $\left\|\varepsilon^{n}-\frac{k}{2} \rho_{1}^{n}+\frac{k^{2}}{8} \rho_{2}^{n}\right\|^{2}=\left\|\varepsilon^{n}\right\|^{2}+\frac{k^{2}}{4}\left\|\rho_{1}^{n}\right\|^{2}+\frac{k^{4}}{64}\left\|\rho_{2}^{n}\right\|^{2}$ $-k\left(\varepsilon^{n}, \rho_{1}^{n}\right)+\frac{k^{2}}{4}\left(\varepsilon^{n}, \rho_{2}^{n}\right)-\frac{k^{3}}{8}\left(\rho_{1}^{n}, \rho_{2}^{n}\right)$. Now, by (5.25), $\left(\varepsilon^{n}, \rho_{1}^{n}\right)=\left(\varepsilon^{n},\left(U^{n} \varepsilon^{n}\right)_{x}\right)$ $=\frac{1}{2}\left(U_{x}^{n} \varepsilon^{n}, \varepsilon^{n}\right)$. Also, by (5.32), (5.25), $\left(\varepsilon^{n}, \rho_{2}^{n}\right)=-\left(\varepsilon_{x}^{n}, U^{n} \rho_{1}^{n}\right)=-\left\|\rho_{1}^{n}\right\|^{2}+$ $\left(U_{x}^{n} \varepsilon^{n}, \rho_{1}^{n}\right),\left(\rho_{1}^{n}, \rho_{2}^{n}\right)=\frac{1}{2}\left(U_{x}^{n} \rho_{1}^{n}, \rho_{1}^{n}\right)$. Therefore, we conclude that

$\left\|\varepsilon^{n}-\frac{k}{2} \rho_{1}^{n}+\frac{k^{2}}{8} \rho_{2}^{n}\right\|^{2}=\left\|\varepsilon^{n}\right\|^{2}+\frac{k^{4}}{64}\left\|\rho_{2}^{n}\right\|^{2}-\frac{k}{2}\left(U_{x}^{n} \varepsilon^{n}, \varepsilon^{n}\right)+\frac{k^{2}}{4}\left(U_{x}^{n} \varepsilon^{n}, \rho_{1}^{n}\right)-\frac{k^{3}}{16}\left(U_{x}^{n} \rho_{1}^{n}, \rho_{1}^{n}\right)$.

Now, by the approximation and inverse properties of $S_{h, 0}^{2}$ we have by (5.25), (5.32) that $\left|\left(U_{x}^{n} \varepsilon^{n}, \varepsilon^{n}\right)\right| \leq C\left\|\varepsilon^{n}\right\|^{2},\left|\left(U_{x}^{n} \varepsilon^{n}, \rho_{1}^{n}\right)\right| \leq C\left\|\varepsilon^{n}\right\|\left\|\rho_{1}^{n}\right\| \leq C h^{-1}\left\|\varepsilon^{n}\right\|^{2},\left|\left(U_{x}^{n} \rho_{1}^{n}, \rho_{1}^{n}\right)\right|$ $\leq C\left\|\rho_{1}^{n}\right\|^{2} \leq C h^{-2}\left\|\varepsilon^{n}\right\|^{2},\left\|\rho_{2}^{n}\right\| \leq C h^{-2}\left\|\varepsilon^{n}\right\|$. Inserting these estimates in (5.44) and recalling that $\mu=k / h^{4 / 3}$ we are led to the inequality $\left\|\varepsilon^{n}-\frac{k}{2} \rho_{1}^{n}+\frac{k^{2}}{8} \rho_{2}^{n}\right\|^{2} \leq$ $\left(1+C k \mu^{3}+C k+C k \lambda+C k \lambda^{2}\right)\left\|\varepsilon^{n}\right\|^{2} \leq\left(1+C_{\mu} k\right)\left\|\varepsilon^{n}\right\|^{2}$, and, hence, to

$$
\left\|\varepsilon^{n}-\frac{k}{2} \rho_{1}^{n}+\frac{k^{2}}{8} \rho_{2}^{n}\right\| \leq\left(1+C_{\mu} k\right)\left\|\varepsilon^{n}\right\|,
$$

where, by $C_{\mu}$ we denote a constant depending polynomially on $\mu$; we have replaced $C_{\lambda}$ 's by $C_{\mu}$ 's since $\lambda=h^{1 / 3} \mu \leq \mu$. We finally obtain from (5.45), (5.43) and (5.31), for $0 \leq n \leq n^{*}$, that

$$
\left\|\varepsilon^{n+1}\right\| \leq\left\|\varepsilon^{n}\right\|+C_{\mu} k\left(\left\|\varepsilon^{n}\right\|+\left\|e^{n}\right\|_{1}\right)+C_{\mu} k\left(k^{2}+h^{3 / 2}\right) .
$$

We now estimate the $\|\cdot\|_{1}$ norm of the various terms in the right-hand side of (5.34). For $0 \leq n \leq n^{*}$, by (5.4), (5.13), (5.35), and (5.38) we have $\| A\left(\left(U^{n, 1} \xi^{n}\right)_{x}\right.$ $\left.-\xi^{n} \xi_{x}^{n}\right)\left\|_{1} \leq C\right\| U^{n, 1} \xi^{n}-\frac{1}{2}\left(\xi^{n}\right)^{2}\|\leq C\| \xi^{n} \|_{1} \leq C\left(\left\|\varepsilon^{n}\right\|+\left\|e^{n}\right\|_{1}\right)$. Similarly, for $0 \leq n \leq n^{*}$, by (5.39),$\left\|A\left(\left(H^{n, 1} \theta^{n}\right)_{x}-\theta^{n} \theta_{x}^{n}\right)\right\|_{1} \leq C\left\|H^{n, 1} \theta^{n}-\frac{1}{2}\left(\theta^{n}\right)^{2}\right\| \leq C_{\lambda}\left\|\theta^{n}\right\|$ $\leq C_{\lambda}\left(\left\|\varepsilon^{n}\right\|+\left\|e^{n}\right\|_{1}\right)$. Therefore, using (5.34), (5.39) and Lemma 5.2 we have for $0 \leq n \leq n^{*}$,

$$
\begin{aligned}
\left\|e^{n+1}\right\|_{1} & \leq\left\|e^{n}\right\|_{1}+C k\left\|\theta^{n}\right\|+C_{\lambda} k\left(\left\|\varepsilon^{n}\right\|+\left\|e^{n}\right\|_{1}\right)+C_{\lambda} k\left(k^{2}+h^{3 / 2}\right) \\
& \leq\left\|e^{n}\right\|_{1}+C_{\lambda} k\left(\left\|\varepsilon^{n}\right\|+\left\|e^{n}\right\|_{1}\right)+C_{\lambda} k\left(k^{2}+h^{3 / 2}\right) .
\end{aligned}
$$

Now adding (5.46) and (5.47), we conclude for $0 \leq n \leq n^{*}$ that $\left\|\varepsilon^{n+1}\right\|+\left\|e^{n+1}\right\|_{1} \leq$ $\left(1+C_{\mu} k\right)\left(\left\|\varepsilon^{n}\right\|+\left\|e^{n}\right\|_{1}\right)+C_{\mu} k\left(k^{2}+h^{3 / 2}\right)$. Using Gronwall's Lemma and taking $h$ sufficiently small contradicts the maximality of $n^{*}$. Hence $n^{*}$ may be taken equal to $M-1$ and it holds that

$$
\left\|\varepsilon^{n}\right\|+\left\|e^{n}\right\|_{1} \leq \exp \left(C_{\mu} T\right)\left(k^{2}+h^{3 / 2}\right), \quad 0 \leq n \leq M,
$$

i.e., that (5.21) is valid; the conclusion of the proposition follows. The (CB) case is entirely similar.

Remark 5.1. The last estimate and Sobolev's inequality imply that $\left\|e^{n}\right\|_{\infty}=O\left(k^{2}+\right.$ $\left.h^{3 / 2}\right)$. Therefore, $\max _{n}\left\|u\left(t^{n}\right)-U_{h}^{n}\right\|_{\infty}=O\left(k^{2}+h^{3 / 2}\right)$.

Remark 5.2. Consider the linearized problem (3.25). In this case, the proof of Proposition 5.2 is considerably simplified and yields $\left\|\varepsilon^{n}\right\|+\left\|e^{n}\right\|_{1} \leq C\left(k^{2}+h^{3 / 2}\right), 0$ $\leq n \leq M$, and the other estimates of Proposition 5.2 without the stability restriction $k=O\left(h^{4 / 3}\right)$. In other words, the linearized system is not stiff. This may also be 
verified by examining the spectrum of the spatial discretization operator of the semidiscrete linearized system: In [6] it is proved that the eigenvalues are purely imaginary and bounded by a constant independent of $h$. No stability restrictions are needed in this case for the other two fully discrete schemes either.

Remark 5.3. Numerical experiments in [6] seem to indicate that the stability condition $k=O\left(h^{4 / 3}\right)$ is also necessary in some sense. The numerical results were stable and accurate when we took $k=h^{4 / 3}$ but led to overflow in finite time when we took $k=h$.

5.3. Fourth-order Runge-Kutta scheme with cubic splines. Our third example is time stepping with the classical, fourth-order accurate four-stage explicit Runge-Kutta scheme, written in the case of the ode $y^{\prime}=f(t, y)$ in the three-step form $y^{n, 1}=y^{n}+\frac{k}{2} f\left(t^{n}+\frac{k}{2}, y^{n}\right), y^{n, 2}=y^{n}+\frac{k}{2} f\left(t^{n}+\frac{k}{2}, y^{n, 1}\right), y^{n, 3}=y^{n}+$ $k f\left(t^{n}+k, y^{n, 2}\right), y^{n+1}=y^{n}+k\left(\frac{1}{6} f\left(t^{n}, y^{n}\right)+\frac{1}{3} f\left(t^{n}+\frac{k}{2}, y^{n, 1}\right)+\frac{1}{3} f\left(t^{n}+\frac{k}{2}, y^{n, 2}\right)\right.$ $\left.+\frac{1}{6} f\left(t^{n}+k, y^{n, 3}\right)\right)$. We will couple this time-stepping scheme with a space discretization that uses cubic splines on a uniform mesh on $[0,1]$. We consider only the case of ( $(\mathrm{SCB})$, that of ( $\mathrm{CB})$ being analogous. As usual we let $M$ be a positive integer, $k=T / M$ and $t^{n}=n k$, for $n=0,1, \ldots, M$. For $0 \leq n \leq M$ we seek $\left(H_{h}^{n}, U_{h}^{n}\right) \in S_{h}^{4} \times S_{h, 0}^{4}$ approximations of $\eta\left(t^{n}\right), u\left(t^{n}\right)$, and for $0 \leq n \leq M-1$ $\left(H_{h}^{n, j}, U_{h}^{n, j}\right) \in S_{h}^{4} \times S_{h, 0}^{4}, j=1,2,3$, such that for $0 \leq n \leq M-1$,

$$
\begin{aligned}
H_{h}^{n, j}-H_{h}^{n}+k a_{j} P\left(U_{h}^{n, j-1}+\frac{1}{2} H_{h}^{n, j-1} U_{h}^{n, j-1}\right)_{x} & =0, \\
U_{h}^{n, j}-U_{h}^{n}+k a_{j} A\left(H_{h x}^{n, j-1}+\frac{3}{2} U_{h}^{n, j-1} U_{h x}^{n, j-1}+\frac{1}{2} H_{h}^{n, j-1} H_{h x}^{n, j-1}\right) & =0,
\end{aligned}
$$

for $j=1,2,3$ and

$$
\begin{array}{r}
H_{h}^{n+1}-H_{h}^{n}+k P\left[\sum_{j=1}^{4} b_{j}\left(U_{h}^{n, j-1}+\frac{1}{2} H_{h}^{n, j-1} U_{h}^{n, j-1}\right)\right]_{x}=0, \\
U_{h}^{n+1}-U_{h}^{n}+k A\left[\sum_{j=1}^{4} b_{j}\left(H_{h x}^{n, j-1}+\frac{3}{2} U_{h x}^{n, j-1} U_{h}^{n, j-1}+\frac{1}{2} H_{h x}^{n, j-1} H_{h}^{n, j-1}\right)\right]=0,
\end{array}
$$

where $H_{h}^{n, 0}=H_{h}^{n}, U_{h}^{n, 0}=U_{h}^{n}, a_{1}=a_{2}=1 / 2, a_{3}=1, b_{1}=b_{4}=1 / 6, b_{2}=b_{3}=$ $1 / 3$, and

$$
H_{h}^{0}=I_{h} \eta_{0}, \quad U_{h}^{0}=R_{h} u_{0} .
$$

(We have denoted again by $P: L^{2} \rightarrow S_{h}^{4}$ the $L^{2}$-projection operator, defined $A$ : $L^{2} \rightarrow S_{h, 0}^{4}$ by (5.4) posed on $S_{h, 0}^{4}, I_{h}$ as the interpolant in $S_{h}^{4}$ and $R_{h}$ as the elliptic projection onto $S_{h, 0}^{4}$.) The stability and convergence of the fully discrete scheme require that $k / h$ is sufficiently small. The proof is long and technical and may be found in [6]. Here we just state the final result.

Proposition 5.3. Suppose that the solution $(\eta, u)$ of $(\overline{\mathrm{SCB}})$ is sufficiently smooth on $[0, T]$. Let $\lambda=k / h$ and $\left(H_{h}^{n}, U_{h}^{n}\right)$ be the solution of (5.48)-(5.49). Then, there exists a positive constant $\lambda_{0}$ and a constant $C$ independent of $k$ and $h$, such that for $\lambda \leq \lambda_{0}$,

$$
\begin{gathered}
\max _{0 \leq n \leq M}\left\|\eta\left(t^{n}\right)-H_{h}^{n}\right\| \leq C\left(k^{4}+h^{3.5} \sqrt{\ln 1 / h}\right), \\
\max _{0 \leq n \leq M}\left\|u\left(t^{n}\right)-U_{h}^{n}\right\|_{1} \leq C\left(k^{4}+h^{3}\right) .
\end{gathered}
$$

An entirely similar result holds for (CB). 


\section{Numerical EXPERIMENTS}

In this section we present the results of some numerical experiments that we performed in order to illustrate and explore further theoretical results that were proved in the previous sections. The interested reader may find more numerical results in 6 .

TABLE 6.1. Errors and orders of convergence. (CB) system, standard Galerkin semidiscretization with piecewise linear, continuous functions on a uniform mesh.

\begin{tabular}{|c|c|c|c|c|c|c|c|c|}
\hline & \multicolumn{4}{|c|}{$L^{2}-$ errors } & \multicolumn{4}{c|}{$H^{1}-$ errors } \\
\hline$N$ & $\eta$ & order & $u$ & order & $\eta$ & order & $u$ & order \\
\hline 80 & $0.6849(-2)$ & & $0.4259(-4)$ & & $0.1776(+1)$ & & $0.1192(-1)$ & \\
\hline 160 & $0.2454(-2)$ & 1.481 & $0.1051(-4)$ & 2.019 & $0.1277(+1)$ & 0.476 & $0.5880(-2)$ & 1.019 \\
\hline 240 & $0.1342(-2)$ & 1.488 & $0.4652(-5)$ & 2.010 & $0.1049(+1)$ & 0.486 & $0.3902(-2)$ & 1.011 \\
\hline 320 & $0.8738(-3)$ & 1.492 & $0.2611(-5)$ & 2.007 & 0.9109 & 0.490 & $0.2920(-2)$ & 1.008 \\
\hline 400 & $0.6261(-3)$ & 1.494 & $0.1669(-5)$ & 2.006 & 0.8161 & 0.492 & $0.2333(-2)$ & 1.006 \\
\hline 480 & $0.4767(-3)$ & 1.495 & $0.1158(-5)$ & 2.005 & 0.7459 & 0.494 & $0.1942(-2)$ & 1.005 \\
\hline 520 & $0.4230(-3)$ & 1.495 & $0.9864(-6)$ & 2.004 & 0.7170 & 0.494 & $0.1792(-2)$ & 1.004 \\
\hline
\end{tabular}

6.1. Standard Galerkin semidiscretization with piecewise linear, continuous functions. We first consider the case of uniform mesh with $h=1 / N$ on $[0,1]$. Table 6.1 shows the errors and the associated rates of convergence for increasing $N$ in the $L^{2}$ - and $H^{1}$-norms at $T=1$ of the approximation to the (CB) system with suitable right-hand side and initial conditions so that its exact solution is given by

$$
\eta(x, t)=\exp (2 t)(\cos (\pi x)+x+2), \quad u(x, t)=\exp (-x t) x \sin (\pi x) .
$$

The system was integrated up to $T=1$ with the classical, four-stage, fourth-order, explicit Runge-Kutta method (henceforth referred to as RK4) of section 5.3, using a time step $k=h / 10$. We checked that temporal error of the discretization was very small compared to the spatial error, so that the errors and rates of convergence shown are essentially those of the semidiscrete problem (2.7), (3.2). The table suggests that the $L^{2}$ rates of convergence of $\eta$ and $u$ approach $3 / 2$ and 2, respectively, and that $\left\|u-u_{h}\right\|_{1}=O(h)$, thus confirming the estimates of Theorem 3.1. It also suggests that $\left\|\eta-\eta_{h}\right\|_{1}=O\left(h^{1 / 2}\right)$. The convergence rates for the analogous problem for the $(\mathrm{SCB})$ system were essentially the same. The observed maximum norm errors were $\left\|\eta-\eta_{h}\right\|_{\infty}=O(h),\left\|u-u_{h}\right\|_{\infty}=O\left(h^{2}\right) ;$ cf. [6].

We then integrated a suitably nonhomogeneous version of the (CB) system with exact solution given by

(6.2) $\eta(x, t)=\exp (2 t)(\cos (\pi x)+x+2), \quad u(x, t)=\exp (x t)\left(\sin (\pi x)+x^{3}-x^{2}\right)$,

on the quasiuniform mesh on $[0,1]$ given by $h_{2 i-1}=1.2 \Delta x, h_{2 i}=0.8 \Delta x, 1 \leq i$ $\leq N / 2$, where $h_{i}=x_{i+1}-x_{i}$ and $\Delta x=1 / N$. (The RK4 scheme was used for time-stepping with $k=\Delta x / 10$.) We integrated the system up to $T=0.4$ starting with the $L^{2}$-projections of $\eta_{0}$ and $u_{0}$ on the finite element subspaces. (We checked that the temporal error was much smaller than the spatial error.) Table 6.2(a) shows the $L^{2}$-errors for $\eta$ and $u$ and the associated rates of convergence at $T=$ 0.4. The data strongly suggest that $\left\|\eta-\eta_{h}\right\|=O(h)$ and $\left\|u-u_{h}\right\|=O\left(h^{2}\right)$, thus confirming the relevant theoretical result for $\eta$ (cf. Proposition 2.1) and supporting the conjecture that the $L^{2}$ rate of convergence for $u$ is actually equal to 2 even in 
TABLE 6.2. $L^{2}$-errors and orders of convergence. (CB system, standard Galerkin semidiscretization with piecewise linear, continuous functions on a quasiuniform mesh with $\frac{\max h_{i}}{\min h_{i}}=1.5$ (a), $\frac{\max h_{i}}{\min h_{i}}=150(\mathrm{~b})$

\begin{tabular}{|c|c|c|c|c|c|c|c|c|c|}
\hline \multicolumn{9}{|c|}{$(\mathrm{a})$} & \multicolumn{5}{c|}{ (b) } \\
\hline$N$ & $\eta$ & order & $u$ & order & $N$ & $\eta$ & order & $u$ & order \\
\hline 80 & $0.1277(-1)$ & & $0.7432(-4)$ & & 120 & $0.1942(-1)$ & & $0.1899(-3)$ & \\
\hline 160 & $0.6383(-2)$ & 1.000 & $0.1858(-4)$ & 2.000 & 200 & $0.1155(-1)$ & 1.017 & $0.6834(-4)$ & 2.000 \\
\hline 240 & $0.4258(-2)$ & 0.999 & $0.8259(-5)$ & 2.000 & 240 & $0.9600(-2)$ & 1.014 & $0.4745(-4)$ & 2.001 \\
\hline 320 & $0.3194(-2)$ & 0.999 & $0.4646(-5)$ & 2.000 & 320 & $0.7176(-2)$ & 1.012 & $0.2669(-4)$ & 2.001 \\
\hline 400 & $0.2556(-2)$ & 0.999 & $0.2973(-5)$ & 2.000 & 360 & $0.6371(-2)$ & 1.010 & $0.2109(-4)$ & 2.000 \\
\hline 480 & $0.2131(-2)$ & 0.999 & $0.2065(-5)$ & 2.000 & 400 & $0.5729(-2)$ & 1.009 & $0.1708(-4)$ & 2.001 \\
\hline
\end{tabular}

TABLE 6.3. Errors and orders of convergence. (SCB system, standard Galerkin semidiscretization with cubic splines on a uniform mesh.

\begin{tabular}{|c|c|c|c|c|c|c|c|c|}
\hline & \multicolumn{4}{|c|}{$L^{2}-$ errors } & \multicolumn{4}{|c|}{$H^{1}$ - errors } \\
\hline$N$ & $\eta$ & order & $u$ & order & $\eta$ & order & $u$ & order \\
\hline 80 & $0.7178(-7)$ & & $0.5062(-8)$ & & $0.2215(-4)$ & & $0.2540(-5)$ & \\
\hline 160 & $0.6393(-8)$ & 3.489 & $0.3178(-9)$ & 3.994 & $0.3829(-5)$ & 2.533 & $0.3190(-6)$ & 2.993 \\
\hline 240 & $0.1553(-8)$ & 3.490 & $0.6288(-10)$ & 3.996 & $0.1379(-5)$ & 2.519 & $0.9467(-7)$ & 2.996 \\
\hline 320 & $0.5691(-9)$ & 3.490 & $0.1986(-10)$ & 4.006 & $0.6699(-6)$ & 2.510 & $0.3997(-7)$ & 2.997 \\
\hline 400 & $0.2612(-9)$ & 3.489 & $0.8106(-11)$ & 4.016 & $0.3831(-6)$ & 2.505 & $0.2047(-7)$ & 2.998 \\
\hline 480 & $0.1382(-9)$ & 3.490 & $0.4011(-11)$ & 3.859 & $0.2428(-6)$ & 2.501 & $0.1185(-7)$ & 2.998 \\
\hline 520 & $0.1046(-9)$ & 3.488 & $0.2840(-11)$ & 4.315 & $0.1988(-6)$ & 2.499 & $0.9323(-8)$ & 2.998 \\
\hline
\end{tabular}

the case of the nonlinear problem; recall that the technique of proof of Theorem 3.1 in the case of a quasiuniform mesh gives a pessimistic bound of $O\left(h^{3 / 2}\right)$ for $\left\|u-u_{h}\right\|$; cf. Remark 3.3. These results are confirmed by the rates shown in Table 6.2(b), which was obtained by integrating the same problem with the same method on the quasiuniform mesh on $[0,1]$ with $h_{10 i-9}=0.02 \Delta x, h_{10 i-8}=0.05 \Delta x, h_{10 i-7}$ $=0.08 \Delta x, h_{10 i-6}=0.35 \Delta x, h_{10 i-5}=0.5 \Delta x, h_{10 i-4}=h_{10 i-3}=\Delta x, h_{10 i-2}=$ $h_{10 i-1}=2 \Delta x, h_{10 i}=3 \Delta x, 1 \leq i \leq N / 10$, and $k=\Delta x / 10$. Similar results were obtained for the (SCB) system.

6.2. Semidiscretization with cubic splines. We considered the nonhomogeneous (SCB) system and we discretized it on a uniform mesh using cubic splines for the spatial discretization and RK4 in time with time step $k=h / 10$, for which the temporal discretization error was negligible in comparison with the spatial error. We took a suitable right-hand side so that the exact solution of the system was given by (6.2). The errors and orders of convergence produced by this numerical experiment are shown in Table 6.3. The rates are close to the theoretical predictions of Theorem 4.1. The table suggests that the $L^{2}$ rate of convergence for $\eta$ is slightly less than 3.5, while that for $u$ is close to four. It further suggests that $\left\|\eta-\eta_{h}\right\|_{1}=O\left(h^{2.5}\right),\left\|u-u_{h}\right\|_{1}=O\left(h^{3}\right)$ (agreeing with the second estimate of (i) of Theorem 4.1). We also mention that the $L^{\infty}$ errors were measured to be $\left\|\eta-\eta_{h}\right\|_{\infty}=O\left(h^{3}\right)$ and $\left\|u-u_{h}\right\|_{\infty}=O\left(h^{4}\right)$, and that the convergence rates from a similar experiment with (CB) were practically the same; cf. 6]. When we plotted the quantity $\kappa:=\left\|\eta-\eta_{h}\right\| /\left(h^{3.5} \sqrt{\ln 1 / h}\right)$ as a function of $N=1 / h$ we observed 
that $\kappa$ apparently approaches a constant close to 0.13 as $N$ grows, which seems to be consistent with the presence of a slow-varying modulation of $h^{3.5}$ as $h \rightarrow$ 0 . We close this paragraph with a remark on the "effect of the boundary" on the error estimates of Theorem 4.1. The proofs of Lemma 4.4 and Theorem 4.1 suggest that the accuracy of $\psi$ in Lemma 4.4 and, e.g., that of $\left\|\eta-\eta_{h}\right\|$ in Theorem 4.1 degenerate near the boundary of the interval. This is consistent with the results of the following numerical experiment. We integrated in time the (SCB system on $[0,1]$ with suitable right-hand side and initial conditions so that the wave elevation is given by the travelling Gaussian profile $\eta(x, t)=0.5 \exp \left[-144(x-0.5-0.2 t)^{2}\right]$ and the velocity by $u(x, t)=6(\sqrt{\eta+1}-1) x(x-1)$. The support of the initial $\eta$-profile is effectively contained in the interval $[0.3,0.5]$ and the wave moves to the right and starts crossing the boundary at $x=1$ at about $t=1.5$ (see Figure 6.1). In Table 6.4 we show the $L^{2}$ errors of $\eta$, as $N=1 / h$ increases, at the temporal instances $t=1.0,1.5,2.0$ and 2.5. The rates of convergence are practically equal to four up to $t=1.5$ but as $\eta$ becomes nonzero at the boundary they fall to a value consistent with the first inequality of $(i)$ of Theorem 4.1.

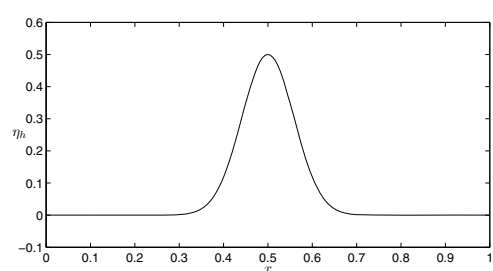

(a) $\eta_{h}$ at $t=0.0$

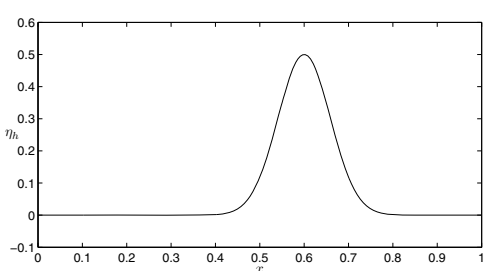

(b) $\eta_{h}$ at $t=0.5$

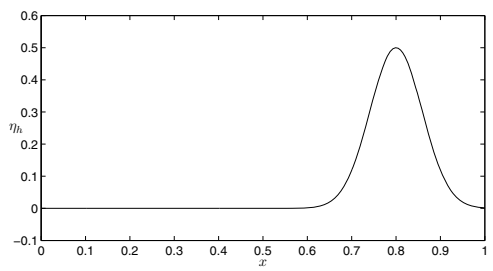

(c) $\eta_{h}$ at $t=1.5$

Figure 6.1. Travelling Gaussian $\eta$-profile. Nonhomogeneous (SCB) system.

TABLE 6.4. $L^{2}$-errors of $\eta$ and orders of convergence. Example of Figure 6.1.

\begin{tabular}{|c|c|c|c|c|c|c|c|c|}
\hline time & \multicolumn{2}{|c|}{1.0} & \multicolumn{2}{|c|}{1.5} & \multicolumn{2}{c|}{2.0} & \multicolumn{2}{c|}{2.5} \\
\hline$N$ & $L^{2}$-error & order & $L^{2}$-error & order & $L^{2}$-error & order & $L^{2}$-error & order \\
\hline 250 & $1.0661(-08)$ & & $1.3596(-08)$ & & $1.5924(-08)$ & & $1.9906(-08)$ & \\
\hline 500 & $6.6223(-10)$ & 4.009 & $8.4585(-10)$ & 4.007 & $1.0596(-09)$ & 3.910 & $1.7594(-09)$ & 3.500 \\
\hline 750 & $1.3067(-10)$ & 4.003 & $1.6706(-10)$ & 4.000 & $2.2223(-10)$ & 3.852 & $4.2637(-10)$ & 3.496 \\
\hline 1000 & $4.1350(-11)$ & 4.000 & $5.2838(-11)$ & 4.001 & $7.4176(-11)$ & 3.814 & $1.5595(-10)$ & 3.496 \\
\hline 1250 & $1.6922(-11)$ & 4.004 & $2.1710(-11)$ & 3.986 & $3.1966(-11)$ & 3.772 & $7.1471(-11)$ & 3.497 \\
\hline 1500 & $8.1703(-12)$ & 3.994 & $1.0554(-11)$ & 3.956 & $1.6213(-11)$ & 3.724 & $3.7803(-11)$ & 3.493 \\
\hline
\end{tabular}




\section{REFERENCES}

1. K. Adamy, Existence of solutions for a Boussinesq system on the half line and on a finite interval, Discrete and Continuous Dynamical Systems-Series A 29(2011), 25-49. MR2725279 (2011j:35158)

2. C.J. Amick, Regularity and uniqueness of solutions to the Boussinesq system of equations, J. Differential Equations 54(1984), 231-247. MR757294 (86a:35120)

3. D.C. Antonopoulos, The Boussinesq system of equations: Theory and numerical analysis, Ph.D. Thesis, University of Athens, 2000 (In Greek).

4. D.C. Antonopoulos and V.A. Dougalis, Numerical solution of the "classical" Boussinesq system, Math. and Computers in Simulation 82(2012), 984-1007. MR2903340

5. D.C. Antonopoulos and V.A. Dougalis, Numerical approximations of Boussinesq systems, in Proceedings of the $5^{\text {th }}$ International Conference on Mathematical and Numerical Aspects of Wave Propagation, A. Bermudez et al., eds, SIAM, Philadelphia 2000, pp. 265-269. MR 1785905

6. D.C. Antonopoulos and V.A. Dougalis, Notes on error estimates for Galerkin approximations of the "classical" Boussinesq system and related hyperbolic problems, arXiv:1008.4248.

7. D.C. Antonopoulos, V.A. Dougalis, and D.E. Mitsotakis, Initial-boundary-value problems for the Bona-Smith family of Boussinesq systems, Adv. Differential Equations 14(2009), 27-53. MR2478928(2009k:35250)

8. D.C. Antonopoulos, V.A. Dougalis, and D.E. Mitsotakis, Numerical solution of Boussinesq systems of the Bona-Smith family, Appl. Numer. Math. 60(2010), 314-336. MR2607793 (2011d:65270)

9. D.C. Antonopoulos, V.A. Dougalis, and D.E. Mitsotakis, Galerkin approximations of periodic solutions of Boussinesq systems, Bull. Greek Math. Soc. 57(2010), 13-30.

10. J.L. Bona and M. Chen, A Boussinesq system for two-way propagation of nonlinear dispersive waves, Phys. D 116(1998), 191-224. MR.1621920 (99f:76021)

11. J.L. Bona, M. Chen, and J.-C. Saut, Boussinesq equations and other systems for smallamplitude long waves in nonlinear dispersive media: I. Derivation and linear theory, J. Nonlinear Sci. 12(2002), 283-318. MR1915939 (2003k:35193)

12. J.L. Bona, M. Chen, and J.-C. Saut, Boussinesq equations and other systems for smallamplitude long waves in nonlinear dispersive media : II. The nonlinear theory, Nonlinearity 17(2004), 925-952. MR2057134(2005k:35350)

13. J.L. Bona, T. Colin, and D. Lannes, Long wave approximations for water waves, Arch. Ration. Mech. Anal. 178(2005), 373-410. MR2196497 (2007a:76012)

14. J.L. Bona, V.A. Dougalis, and D.E. Mitsotakis, Numerical solution of KdV-KdV systems of Boussinesq equations: I. The numerical scheme and generalized solitary waves, J. Math. Comp. Simul. 74(2007), 214-228. MR2307859 (2009b:65246)

15. J.L. Bona, V.A. Dougalis, and D.E. Mitsotakis, Numerical solution of KdV-KdV systems of Boussinesq equations: II. Generation and evolution of radiating solitary waves, Nonlinearity 21(2008), 2825-2848. MR2461042(2009i:65165)

16. V.A. Dougalis, A. Duran, M.A. Lopez-Marcos, and D.E. Mitsotakis, A numerical study of the stability of solitary waves of the Bona-Smith system, J. Nonlinear Sci. 17(2007), 329-345. MR2371147(2008k:35404)

17. J. Douglas, Jr., T. Dupont, and L. Wahlbin, Optimal $L^{\infty}$ error estimates for Galerkin approximations to solutions of two-point boundary value problems, Math. Comp. 29(1975), 475-483. MR0371077(51:7298)

18. T. Dupont, Galerkin methods for first order hyperbolics: an example, SIAM J. Numer. Anal. 10(1973), 890-899. MR0349046 (50:1540)

19. A.S. Fokas and B. Pelloni, Boundary value problems for Boussinesq type systems, Math. Phys. Anal. Geom. 8(2005), 59-96. MR2136653 (2006i:35312)

20. B. Pelloni, Spectral methods for the numerical solution of nonlinear dispersive wave equations, Ph.D. Thesis, Yale University, 1996. MR2695225

21. B. Pelloni and V.A. Dougalis, Numerical modelling of two-way propagation of non-linear dispersive waves, J. Math. Comput. Simul. 55(2001),595-606. MR1831398

22. D.H. Peregrine, Calculations of the development of an undular bore, J. Fluid Mech. 25(1966), 321-331.

23. D.H. Peregrine, Long waves on a beach, J. Fluid Mech. 27(1967), 815-827. 
24. D.H. Peregrine, Equations for water waves and the approximations behind them, in Waves on Beaches and Resulting Sediment Transport, R.E. Meyer ed., Academic Press, New York 1972, pp. 95-121.

25. M.E. Schonbeck, Existence of solutions for the Boussinesq system of equations, J. Differential Equations 42(1981), 325-352. MR639225 (83b:35151)

26. R. Schreiber, Finite element methods of high-order accuracy for singular two-point boundary value problems with nonsmooth solutions, SIAM J. Numer. Anal. 17(1980), 547-566. MR584730 (82b:65139)

27. V. Thomée, Galerkin Finite Element Methods for Parabolic Problems, Springer-Verlag, Berlin-Heidelberg 1997. MR1479170 (98m:65007)

28. L.B. Wahlbin, Superconvergence in Galerkin Finite Element Methods, Lecture Notes in Mathematics v. 1605, Springer-Verlag, Berlin-Heidelberg 1995. MR1439050 (98j:65083)

29. G.B. Whitham, Linear and Nonlinear Waves, Wiley, New York 1974. MR0483954 (58:3905)

Department of Mathematics, University of Athens, 15784 Zographou, Greece

E-mail address: antonod@math.uoa.gr

Department of Mathematics, University of Athens, 15784 Zographou, Greece - And Institute of Applied and Computational Mathematics, FORTH, 70013 Heraklion, Greece E-mail address: doug@math.uoa.gr 